\title{
Molecular and atomic gas in the Local Group galaxy M $33^{\star, \star \star}$
}

P. Gratier ${ }^{1}$, J. Braine ${ }^{1}$, N. J. Rodriguez-Fernandez ${ }^{2}$, K. F. Schuster ${ }^{2}$, C. Kramer $^{3}$, E. M. Xilouris ${ }^{4}$, F. S. Tabatabaei ${ }^{5}$, C. Henkel ${ }^{5}$, E. Corbelli ${ }^{6}$, F. Israel ${ }^{7}$, P. P. van der Werf $^{7}$, D. Calzetti ${ }^{8}$, S. Garcia-Burillo ${ }^{9}$, A. Sievers ${ }^{3}$, F. Combes ${ }^{10}$,

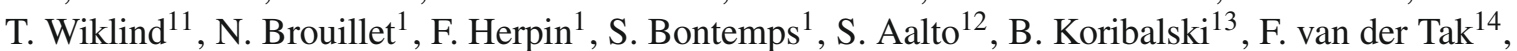
M. C. Wiedner ${ }^{10}$, M. Röllig ${ }^{15}$, and B. Mookerjea ${ }^{16}$

\footnotetext{
${ }^{1}$ Laboratoire d'Astrophysique de Bordeaux, Université de Bordeaux, OASU, CNRS/INSU, 33271 Floirac, France e-mail: gratier@obs.u-bordeaux1.fr

2 IRAM, 300 rue de la piscine, 38406 Saint-Martin d'Hères, France

3 Instituto Radioastronomia Milimetrica (IRAM), Av. Divina Pastora 7, Nucleo Central, 18012 Granada, Spain

${ }^{4}$ Institute of Astronomy \& Astrophysics, National Observatory of Athens, I. Metaxa \& V. Pavlou, P. Penteli, 15236 Athens, Greece

5 Max-Plank-Institut für Radioastronomy (MPIfR), Auf dem Hügel 69, 53121 Bonn, Germany

6 INAF - Osservatorio Astrofisico di Arcetri, LE Fermi, 5, 50125 Firenze, Italy

7 Leiden Observatory, Leiden University, PO Box 9513, 2300 RA Leiden, The Netherlands

8 Department of Astronomy - LGRT, University of Massachusetts - Amherst, 710 north Pleasant Street, Amherst, MA 01002, USA

9 Observatorio Astronomico Nacional (OAN) Observatorio de Madrid, Alfonso XII 3, 28014-Madrid, Spain

${ }_{10}$ Observatoire de Paris, LERMA, CNRS, 61 Av. de l'Observatoire, 75014 Paris, France

11 Space Telescope Science Institute, 3700 San Martin Drive, Baltimore, MD 21218, USA

12 Department of Radio and Space Science, Chalmers University of Technology, Onsala Observatory, 43994 Onsala, Sweden

13 CSIRO Astronomy \& Space Science, Australia Telescope National Facility, PO Box 76, Epping NSW 1710, Australia

14 SRON Netherlands Institute for Space Research, Landleven 12, 9747 AD Groningen, The Netherlands

15 1.Physikalisches Institut, Universität zu Köln, Zülpicher Str. 77, 50937 Köln, Germany

16 Department of Astronomy \& Astrophysics, Tata Institute of Fundamental Research, Homi Bhabha Road, Mumbai 400005, India
}

Received 16 March 2010 / Accepted 11 June 2010

\section{ABSTRACT}

\begin{abstract}
We present high-resolution large-scale observations of the molecular and atomic gas in the Local Group galaxy M 33. The observations were carried out using the HEterodyne Receiver Array (HERA) at the $30 \mathrm{~m}$ IRAM telescope in the $\mathrm{CO}(2-1)$ line, achieving a resolution of $12^{\prime \prime} \times 2.6 \mathrm{~km} \mathrm{~s}^{-1}$, enabling individual giant molecular clouds (GMCs) to be resolved. The observed region is 650 square arcminutes mainly along the major axis and out to a radius of $8.5 \mathrm{kpc}$, and covers entirely the $2^{\prime} \times 40^{\prime}$ radial strip observed with the HIFI and PACS Spectrometers as part of the HERM33ES Herschel key program. The achieved sensitivity in main-beam temperature is $20-50 \mathrm{mK}$ at $2.6 \mathrm{~km} \mathrm{~s}^{-1}$ velocity resolution. The $\mathrm{CO}(2-1)$ luminosity of the observed region is $1.7 \pm 0.1 \times 10^{7} \mathrm{~K} \mathrm{~km} \mathrm{~s}^{-1} \mathrm{pc}^{2}$ and is estimated to be $2.8 \pm 0.3 \times 10^{7} \mathrm{~K} \mathrm{~km} \mathrm{~s}^{-1} \mathrm{pc}^{2}$ for the entire galaxy, corresponding to $\mathrm{H}_{2}$ masses of $1.9 \times 10^{8} M_{\odot}$ and $3.3 \times 10^{8} M_{\odot}$ respectively (including $\mathrm{He}$ ), calculated with $N\left(\mathrm{H}_{2}\right) / I_{\mathrm{CO}(1-0)}$ twice the Galactic value due to the half-solar metallicity of M 33 . The H I $21 \mathrm{~cm}$ VLA archive observations were reduced, and the mosaic was imaged and cleaned using the multi-scale task in the CASA software package, yielding a series of datacubes with resolutions ranging from $5^{\prime \prime}$ to $25^{\prime \prime}$. The H I mass within a radius of $8.5 \mathrm{kpc}$ is estimated to be $1.4 \times 10^{9} M_{\odot}$. The azimuthally averaged CO surface brightness decreases exponentially with a scale length of $1.9 \pm 0.1 \mathrm{kpc}$ whereas the atomic gas surface density is constant at $\Sigma_{\mathrm{HI}}=6 \pm 2 M_{\odot} \mathrm{pc}^{-2}$ deprojected to face-on. For an $N\left(\mathrm{H}_{2}\right) / I_{\mathrm{CO}(1-0)}$ conversion factor twice that of the Milky Way, the central kiloparsec $\mathrm{H}_{2}$ surface density is $\Sigma_{\mathrm{H}_{2}}=8.5 \pm 0.2 \mathrm{M}_{\odot} \mathrm{pc}^{-2}$. The star formation rate per unit molecular gas (SF efficiency, the rate of transformation of molecular gas into stars), as traced by the ratio of $\mathrm{CO}$ to $\mathrm{H}_{\alpha}$ and FIR brightness, is constant with radius. The SFE, with a $N\left(\mathrm{H}_{2}\right) / I_{\mathrm{CO}(1-0)}$ factor twice galactic, appears 2-4 times greater than for large spiral galaxies. A morphological comparison of molecular and atomic gas with tracers of star formation is presented showing good agreement between these maps both in terms of peaks and holes. A few exceptions are noted. Several spectra, including those of a molecular cloud situated more than $8 \mathrm{kpc}$ from the galaxy center, are presented.
\end{abstract}

Key words. galaxies: individual: M 33 - Local Group - galaxies: evolution - galaxies: ISM - ISM: clouds - stars: formation

\section{Introduction}

The Local Group galaxies span a broad range in mass, luminosity, morphology, and metallicity. Two large spirals (the Milky Way and M 31) are the centers of two galaxy subgroupings,

^ Figures A.1-A.15 are only available in electronic form at http: //www . aanda.org

$\star \star$ FITS files of the IRAM data are only available at the CDS via anonymous ftp to cdsarc.u-strasbg.fr (130.79.128.5) or via http://cdsarc.u-strasbg.fr/viz-bin/qcat?]/A+A/522/A3 each being surrounded by a large number of dwarf galaxies. In addition, M 31 - the Andromeda Galaxy - has a small spiral companion, M 33 (the Triangulum Galaxy); their separation is approximately 15 degrees, corresponding to $200 \mathrm{kpc}$ (assuming a common distance of $840 \mathrm{kpc}$, Galleti et al. 2004). Gaseous streams are observed between them, indicating tidal interaction (Putman et al. 2009). M 33 provides a means of observing a galaxy morphologically similar to our own but with a mass only a tenth of the Milky Way and factor two lower metallicity (Rosolowsky \& Simon 2008; Magrini et al. 2009). Further 
Table 1. Adopted parameters for M 33.

\begin{tabular}{lr}
\hline \hline$\alpha(\mathrm{J} 2000)$ & $1^{\mathrm{h}} 33^{\mathrm{m}} 50^{\varsigma} .9$ \\
$\delta(\mathrm{J} 2000)$ & $+30^{\circ} 39^{\prime} 39^{\prime \prime}$ \\
Distance & $840 \mathrm{kpc}^{a}$ \\
Optical radius $R_{25}$ & $30.8^{\prime b}$ \\
Inclination & $56^{\circ b}$ \\
Position angle & $22.5^{\circ b}$ \\
Central oxygen abundance & $12+\log (\mathrm{O} / \mathrm{H})=8.4^{c}$ \\
\hline
\end{tabular}

Notes. ${ }^{(a)}$ Galleti et al. (2004); ${ }^{(b)}$ HYPERLEDA (Paturel et al. 2003); (c) Magrini et al. (2009).

evidence of the difference between M 33 and the Milky Way is the large gas fraction and blue stellar colors of the former relative to the latter. M 33 thus represents an environment in which to study the interstellar medium (ISM) and star formation (SF) that cannot be replaced by Galactic observations and where individual GMCs can be resolved to probe their SF activity. It may also be possible to apply what we learn by studying M 33 to the physics of early-universe objects, which share many of the characteristics of M 33.

In this article we present sensitive and high-resolution mapping observations of the CO $J=2 \rightarrow 1$ transition in M 33 in order to study the morphology and dynamics of the molecular component. The total mapped area covers 650 square arcminutes, mainly along the major axis of the galaxy.

A $2^{\prime} \times 40^{\prime}$ wide strip along the major axis (see Fig. 1) was observed to a particularly low noise level of $25 \mathrm{mK}$ at $2.6 \mathrm{~km} \mathrm{~s}^{-1}$ velocity resolution to compare with the sensitive C II Herschel/HIFI and Herschel/PACS spectroscopy observations which will be obtained as part of the HERM33ES Herschel Key Program (Kramer et al. 2010). While the most sensitive and among the highest resolution, these are not the first maps of M 33 in the CO lines. Engargiola et al. (2003) observed the whole of the inner disk (up to about $5 \mathrm{kpc}$ along the major axis) with the BIMA array at 13" resolution; Heyer et al. (2004) observed the inner disk and a small major axis strip at 50" resolution with FCRAO; Rosolowsky et al. (2007) combined the $\mathrm{BIMA}+\mathrm{FCRAO}+\mathrm{NRO}$ data to improve the sensitivity and resolution of the previous maps; and Gardan et al. (2007) observed a rectangle at high sensitivity and $15^{\prime \prime}$ resolution extending from NGC 604 to the $R_{25}$ radius. Table 2 summarizes the characteristics of previous molecular and atomic gas surveys in M 33. This work extends the Gardan et al. (2007) work further north and to the south at higher resolution and sensitivity. Earlier studies of GMCs in M 33 include Wilson et al. (1997) and Rosolowsky et al. (2003) and studies similar to our own of other Local Group galaxies have been made by e.g. Fukui et al. (2008), Israel et al. (2003), Leroy et al. (2006), and Nieten et al. (2006).

As mentioned by Blitz \& Rosolowsky (2006) for M 33 and Leroy et al. (2006) for IC 10 and discussed more extensively by Gardan et al. (2007), the star formation Rate (SFR) per unit $\mathrm{H}_{2}$ mass or star formation efficiency $\left(S F E=S F R / \mathrm{M}\left(\mathrm{H}_{2}\right)\right.$ in $\left.\mathrm{yr}^{-1}\right)$ was found to be up to an order of magnitude higher in these small galaxies than in large spirals. This appears to be the case in distant galaxies as well, given the factor 10 increase in (commoving) SFR density (e.g. Madau et al. 1996; Wilkins et al. 2008). Are there local universe analogs of these distant objects? Is M 33 one of them?

One of the obvious questions is whether the $\mathrm{H}_{2}$ mass has not been underestimated in these subsolar metallicity objects.

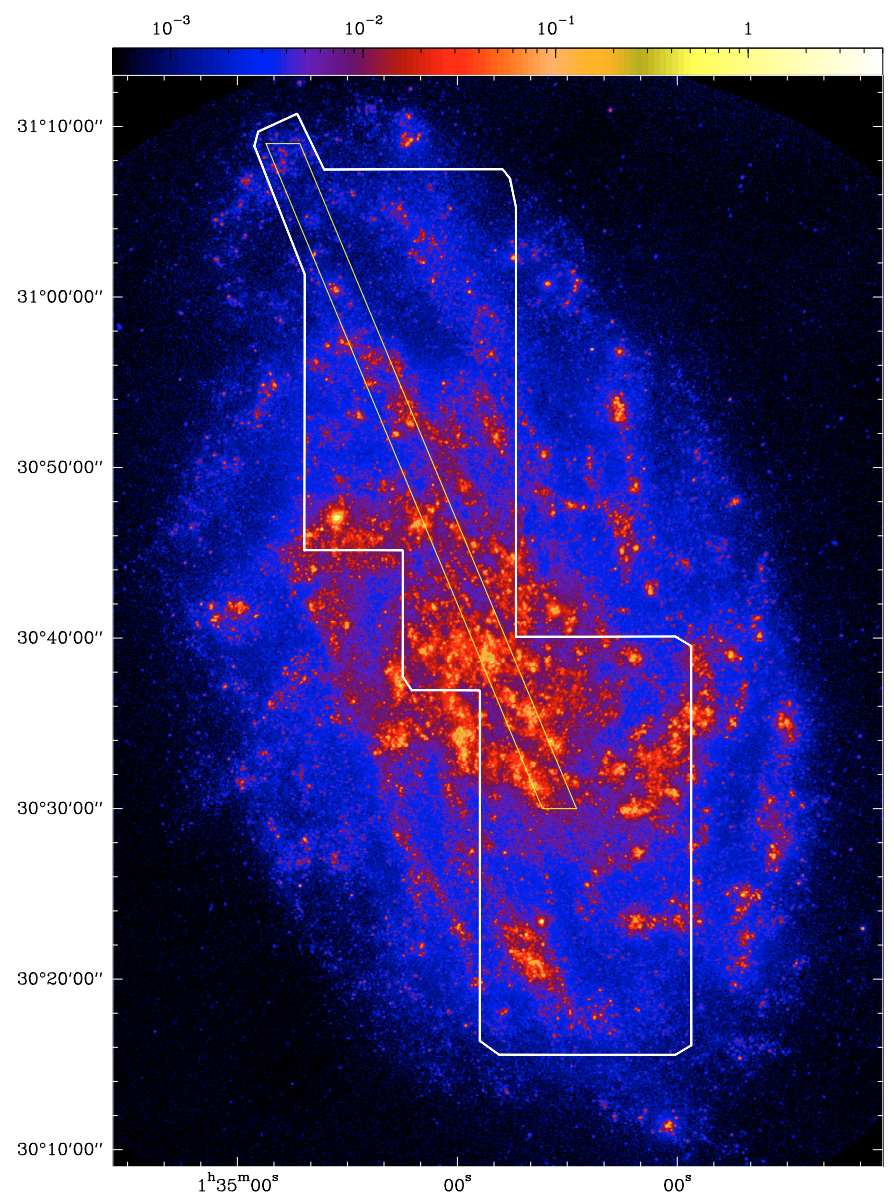

Fig. 1. The Local Group galaxy M 33. This color image shows the GALEX FUV data that trace young stars and dust in the disk through attenuation. Overlaid are the observed fields a) using the IRAM $30 \mathrm{~m}$ Pico Veleta telescope in the $\mathrm{CO}(2-1)$ line (thick white outline) and b) by HIFI/PACS instruments as part of the HERM33ES Herschel Key Program (thin yellow stripe).

The articles using the data presented here and as part of the HERM33ES project will attempt to answer that issue clearly.

Metallicities were lower in the past and $\mathrm{H}_{2}$ production is believed to take place on grain surfaces - therefore $\mathrm{H}$ I to $\mathrm{H}_{2}$ conversion is expected to be less efficient in low-metallicity systems (Krumholz et al. 2009), and this is indeed observed in many systems (e.g. Leroy et al. 2007). The conversion of $\mathrm{H}_{\mathrm{I}}$ to $\mathrm{H}_{2}$ in the intermediate redshift systems would have to be much more efficient than today, generating unusually high molecular fractions in these distant galaxies (contrary to expectations) with less stellar gravity, in order to have a similar efficiency in converting $\mathrm{H}_{2}$ to stars.

Here, we present new maps of $\mathrm{CO}$ and HI. In this first paper, we restrict ourselves to a study of the radial distribution of the molecular gas and infrared surface brightness, of the molecular and atomic gas surface densities and of the SFE, and to a qualitative comparison between the maps of SFR tracers, i.e. the dust maps, and the gas maps. In addition, we will discuss CO spectra in a few selected regions. A series of articles will follow, focusing on at least (i) cloud populations, life cycle, and mass spectrum; (ii) dynamics of the molecular gas and the role of spiral arms; (iii) diffuse $\mathrm{CO}$ emission, after subtracting the clouds identified and; (iv) a more detailed comparison of the SFR - gas surface density relation. 
Table 2. Comparison of past survey characteristics.

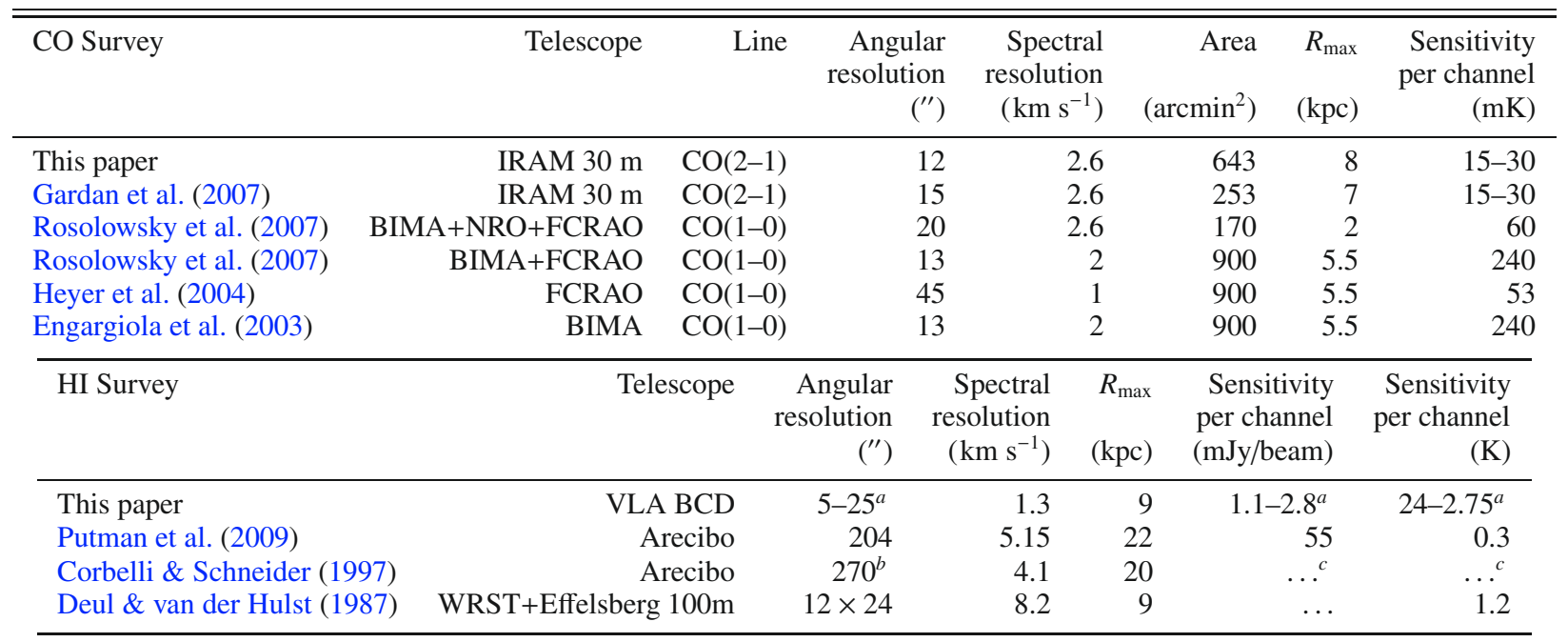

Notes. ${ }^{(a)}$ See Table 3 for details. ${ }^{(b)}$ Undersampled hexagonal grid, the value corresponds to the grid spacing. ${ }^{(c)}$ The authors give an integrated intensity sensitivity value of $1-2 \mathrm{Jy} \mathrm{km} \mathrm{s}^{-1}$ or $5.5-11 \mathrm{~K} \mathrm{~km} \mathrm{~s}^{-1}$.

\section{Observations and data reduction}

\subsection{IRAM CO(2-1) observations}

The observations presented here are a follow-up to the Gardan et al. (2007) mapping of a large part of M 33. All data were taken with the 30 meter telescope run by the Institut de RadioAstronomie Millimétrique $^{1}$ (IRAM) on Pico Veleta near Granada, Spain. The observations were carried out starting in August 2008 and also include the data taken by Gardan et al. (2007).

The IRAM HERA instrument (HEterodyne arRAy), composed of 9 dual-polarization $1.3 \mathrm{~mm}$ receivers (Schuster et al. 2004), was used in On-The-Fly scanning mode. All data are presented in the main beam temperature scale and we have assumed forward and main beam efficiencies of $\eta_{\text {for }}=0.90$ and $\eta_{\mathrm{mb}}=0.49 \pm 0.04$ for the HERA observations, the sensitivity is then $9.6 \mathrm{Jy} / \mathrm{K}$ (Schuster et al. 2004). The scanning speed was 3 arcsec per second of time with dumps every second. Both the VESPA backend, at $1.25 \mathrm{MHz}$ resolution, and the WILMA correlator with $2 \mathrm{MHz}$ channel spacing, were used simultaneously. Reference positions were observed roughly every three minutes and these were taken to be holes in the H I column density outside of the M 33 disk with no visible FIR emission. The average beam size over the nine pixels is $11.7^{\prime \prime}$. The array was tilted by 18.5 degrees, obtaining 7.6 arcsec spacing between the individual pixel tracks, and then shifted in position by 3.8 arcsec in order to obtain full sampling. This was first done for scanning parallel to RA, then parallel to Dec, the array was then rotated by 90 degrees and the procedure was repeated. The array rotation is to have different pixels cover the same regions to improve data homogeneity and reduce striping. For the HIFI low noise major axis strip, additional scans along and perpendicular to the strip direction where acquired. The repetition of independent scans over the same areas also randomizes any pointing errors. Thus, if there are pointing uncertainties of $2^{\prime \prime}$, these contribute negligibly to the overall beam size (through a negligible smearing).

${ }_{1}$ IRAM is supported by INSU/CNRS (France), MPG (Germany) and IGN (Spain).
The part of M 33 presented here comes from the observation of about 5 million spectra.

Reduction was carried out within Gildas ${ }^{2}$ CLASS and GREG software. The intensity of the CO lines is usually small so that emission is not seen in individual spectra, the baseline removal was therefore implemented using a windowing based on the H I data at $17^{\prime \prime}$ presented in Sect. 2.2. In a first step a linear baseline is fitted and subtracted from each individual spectrum not taking into account channels inside a window computed by finding the first channels at a $3 \sigma$ level framing the peak channel of $\mathrm{H} \mathrm{I}$ emission and adding a $40 \mathrm{~km} \mathrm{~s}^{-1}$ buffer on each side of this. A filtering step is then applied to remove spectra with anomalous noise. The actual noise is computed for each spectrum and compared to the theoretical noise computed from the system temperature, integration time and spectral resolution. The spectra with excess noise attributable to poor baselines are removed; this corresponds to about $10 \%$ of the spectra taken. The remaining spectra are then convolved by a Gaussian to obtain a regularly gridded cube of the desired resolution. We then fit and remove a third order polynomial to the spectra corresponding to each of the spatial pixels in the gridded cube this time reducing the velocity buffer described previously to $30 \mathrm{~km} \mathrm{~s}^{-1}$. With this method we have made $\mathrm{CO}$ cubes with spacial resolutions of 12,15 and $25^{\prime \prime}$ and with velocity resolutions of $2.6 \mathrm{~km} \mathrm{~s}^{-1}$.

We compute the $\mathrm{CO}(2-1)$ integrated intensity map using a masking method (Gratier et al. 2010) taking into account H I data, we developed in order to filter out some of the noise present in the observations and increase the sensitivity to low intensity possibly diffuse CO emission. Previous masking methods used masks created from spatially smoothed versions of the original $\mathrm{CO}$ data cubes to filter out regions dominated by noise (Adler et al. 1992; Digel et al. 1996; Loinard et al. 1999). We use the $21 \mathrm{~cm}$ atomic hydrogen data at $17^{\prime \prime} \times 17^{\prime \prime} \times 1.27 \mathrm{~km} \mathrm{~s}^{-1}$ resolution presented in Sect. 2.2 to achieve the same goal, the underlying hypothesis being that molecular gas is unlikely to be present for low enough values of $N(\mathrm{H} \mathrm{I})$ so the corresponding velocity channels can be discarded when computing the integrated intensity $\mathrm{CO}$ map. For each pixel of the $\mathrm{H}$ I cube, we create a binary mask

${ }^{2}$ http://wWw.iram.fr/IRAMFR/GILDAS 


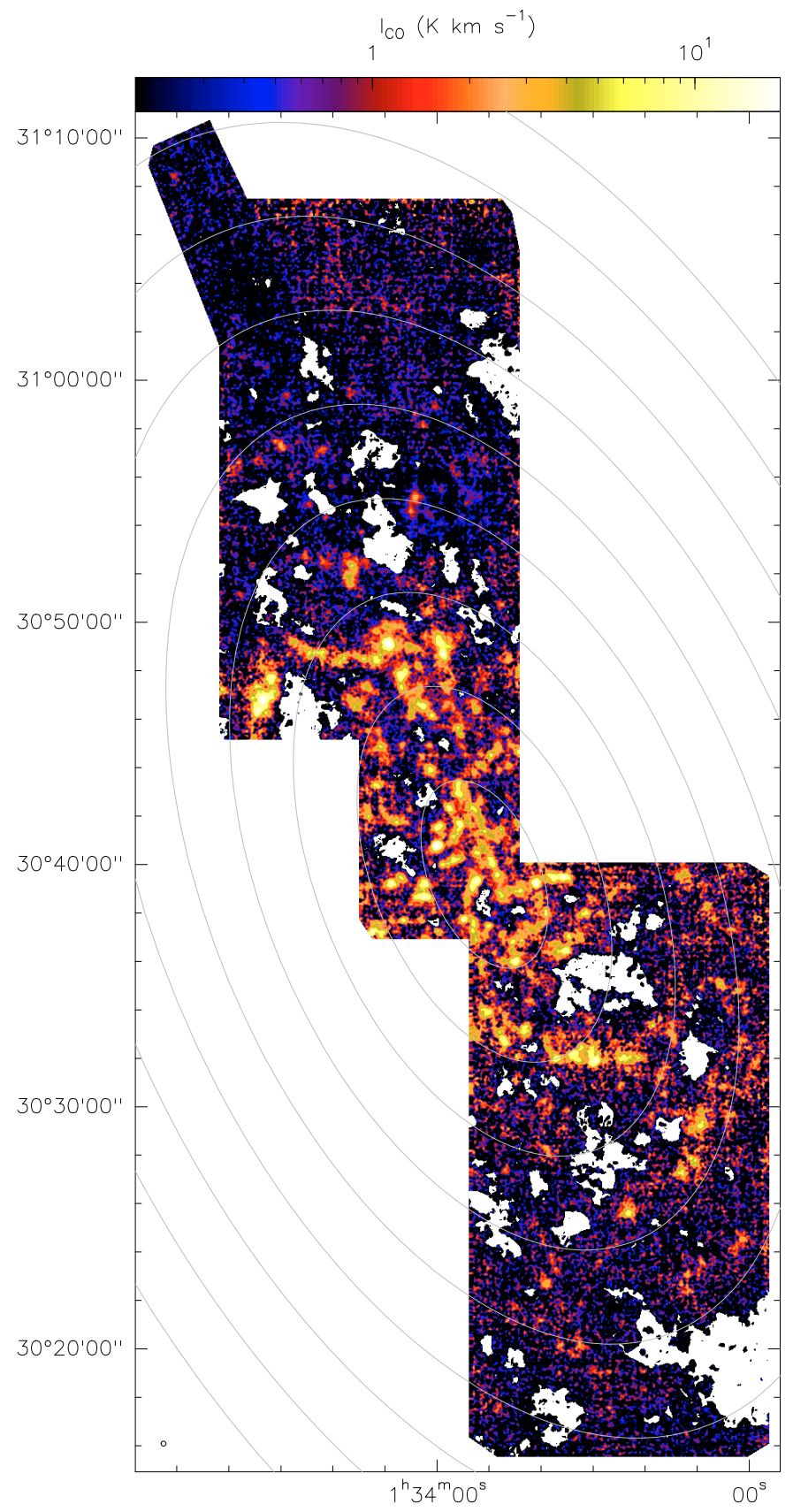

Fig. 2. IRAM CO(2-1) integrated intensity map of the galaxy M 33 at a resolution of $12^{\prime \prime}$. This map was made using the VLA H I cube (17" resolution) as a mask, employing a $10 \mathrm{~K}$ cutoff per channel (see Sect. 2.2). As a consequence, areas without HI emission above that level appear white. Grey ellipses are drawn every kpc using orientation parameters listed in Table 1.

keeping only the velocity range for each pixel corresponding to a H I signal value above a defined threshold in the H I signal. The integrated moment map for the $\mathrm{CO}(2-1)$ data is then computed summing only velocity channels included in the H I mask. Figure 2 shows the integrated intensity map for the $12^{\prime \prime}$ cube using this method and Fig. 3 corresponding noise map at 12" computed from 30 channels free of emission from M 33 (from -80 to $0 \mathrm{~km} \mathrm{~s}^{-1} V_{\mathrm{LSR}}$ ). The result is an increased $\mathrm{S} / \mathrm{N}$ ratio as the channels contributing only noise to the sum are no longer taken into account. The value of the H I signal threshold was chosen at $10 \mathrm{~K}$ which corresponds to $1.9 \sigma$ where $\sigma$ is the rms noise level in

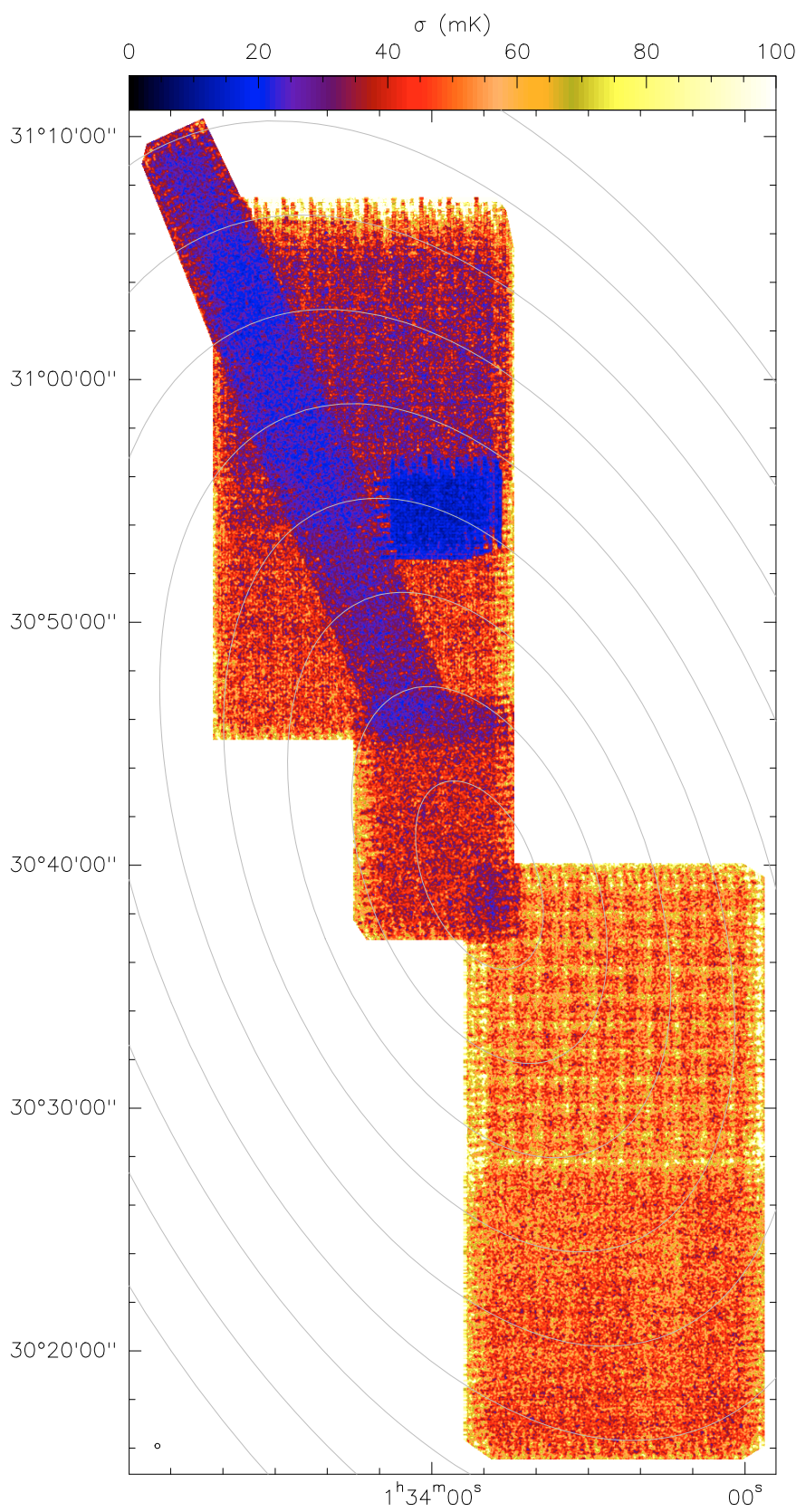

Fig. 3. CO noise map of the $12^{\prime \prime}$ cube computed from 30 channels free of M 33 emission, from -80 to $0 \mathrm{~km} \mathrm{~s}^{-1} V_{\mathrm{LSR}}$.

the $17^{\prime \prime} \mathrm{H}$ I cube. We tested masking values between 5 and $25 \mathrm{~K}$ $(0.9$ to $4.6 \sigma)$ and the total $\mathrm{CO}$ intensity varied by only a few percent. Significantly above or below these values, CO signal is lost or more noise is included. At the $10 \mathrm{~K}$ brightness level, the $\mathrm{H}$ I lines are broad and sample several channels to either side of the rotation curve. Comparing with other techniques of looking for $\mathrm{CO}$, we found no evidence of missing $\mathrm{CO}$ emission by this technique. As an example, the "Lonely Cloud" ( $\alpha=1^{\mathrm{h}} 34^{\mathrm{m}} 16^{\mathrm{s}} .7$, $\delta=+30^{\circ} 59^{\prime} 3^{\prime \prime}$, J2000) (Gardan et al. 2007) in an interarm region not near an H I maximum is included by this method.

\subsection{VLA HI mosaicing}

The HI observations are from archival VLA B, C, and D array data taken as part of projects AT206 and AT268 in 1997 , 


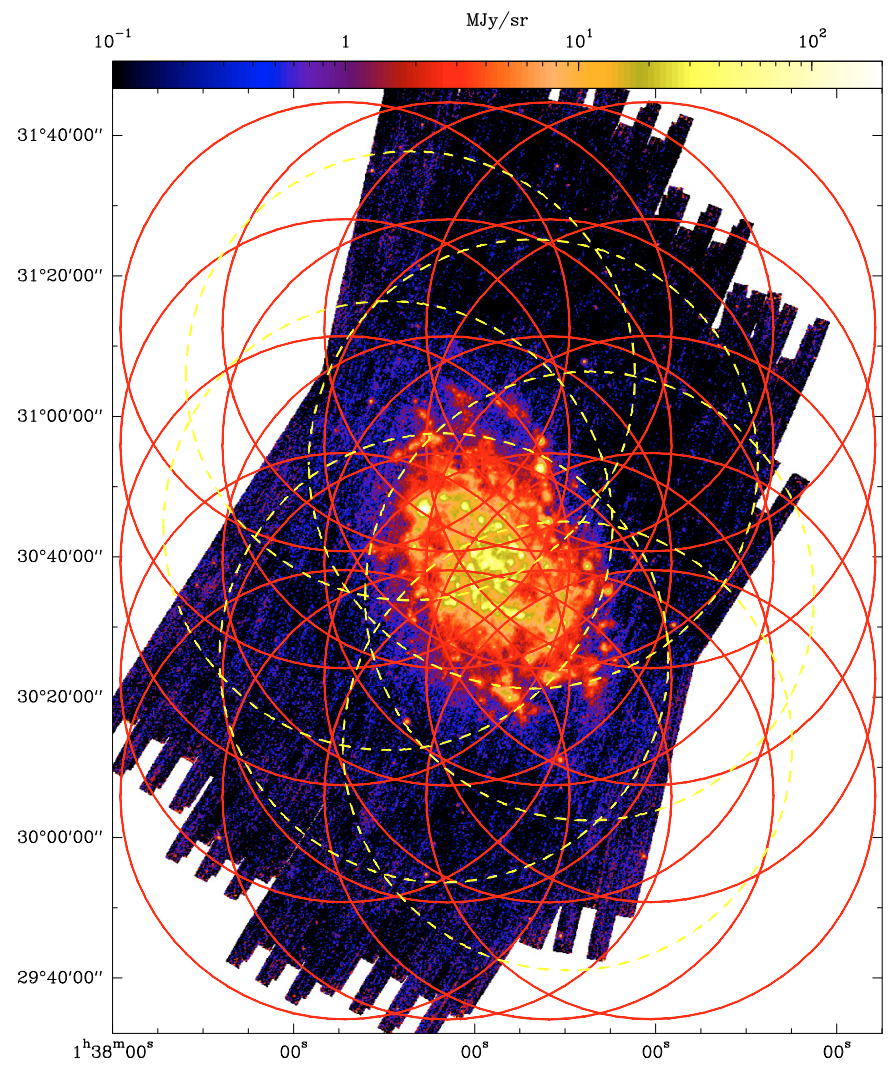

Fig. 4. H I primary beam coverage of M 33 overlaid on the $70 \mu \mathrm{m}$ Spitzer map. VLA D pointings are in red (solid lines) and B/C ones in yellow (dashed lines).

1998 and 2001. At the frequency of the H I line the VLA primary beam is $32^{\prime}$. The mosaic comprises $20 \mathrm{D}$ array pointings on a nearly square grid separated by $16^{\prime}$ and $6 \mathrm{~B}$ and $6 \mathrm{C}$ pointings in a diamond shape covering the entire star forming disk. The primary beam centers of the $\mathrm{B} / \mathrm{C}$ and $\mathrm{D}$ array mosaic are shown in Fig. 4 overlaid on the Spitzer $70 \mu \mathrm{m}$ map - the D array data contains more pointings extending further from the center of M 33. Since our goal is compare the distribution and kinematics of the atomic and molecular gas at high resolution, we focus here on the stellar disk where VLA B-, C- and D-array data are available. Data calibration and imaging were carried out using the 2.4 version of the $\mathrm{CASA}^{3}$ software package. The quasar $3 \mathrm{C} 48\left(\alpha=1^{\mathrm{h}} 37^{\mathrm{m}} 41^{\mathrm{s}} 3, \delta=+33^{\circ} 09^{\prime} 35^{\prime \prime}\right)$ was used as flux, bandpass and phase calibrator (with an adopted flux of $16.04 \mathrm{Jy}$ ). The calibration of the visibilities was done through the following tasks. First the correct flux was applied to the calibrator visibility model using setjy, then frequency bandpass and gain calibrations were computed using the bandpass and gaincal tasks.

The calibration solutions were finally applied to the entire dataset with the use of the applycal task, continuum emission averaged on channels not including line emission from M 33 was subtracted in the $u v$-space using the uvcontsub task. Flagging of bad data was done by hand. The imaging was achieved using the multi-scale clean MSCLEAN algorithm described in Cornwell (2008). This method has several advantages over the classical clean algorithms that use point like sources to model the observed emission. By cleaning several scales simultaneously, it is able to efficiently recover extended emission and eliminates problems of flux correction (Jorsater \& van Moorsel 1995),

3 http://casa.nrao.edu/

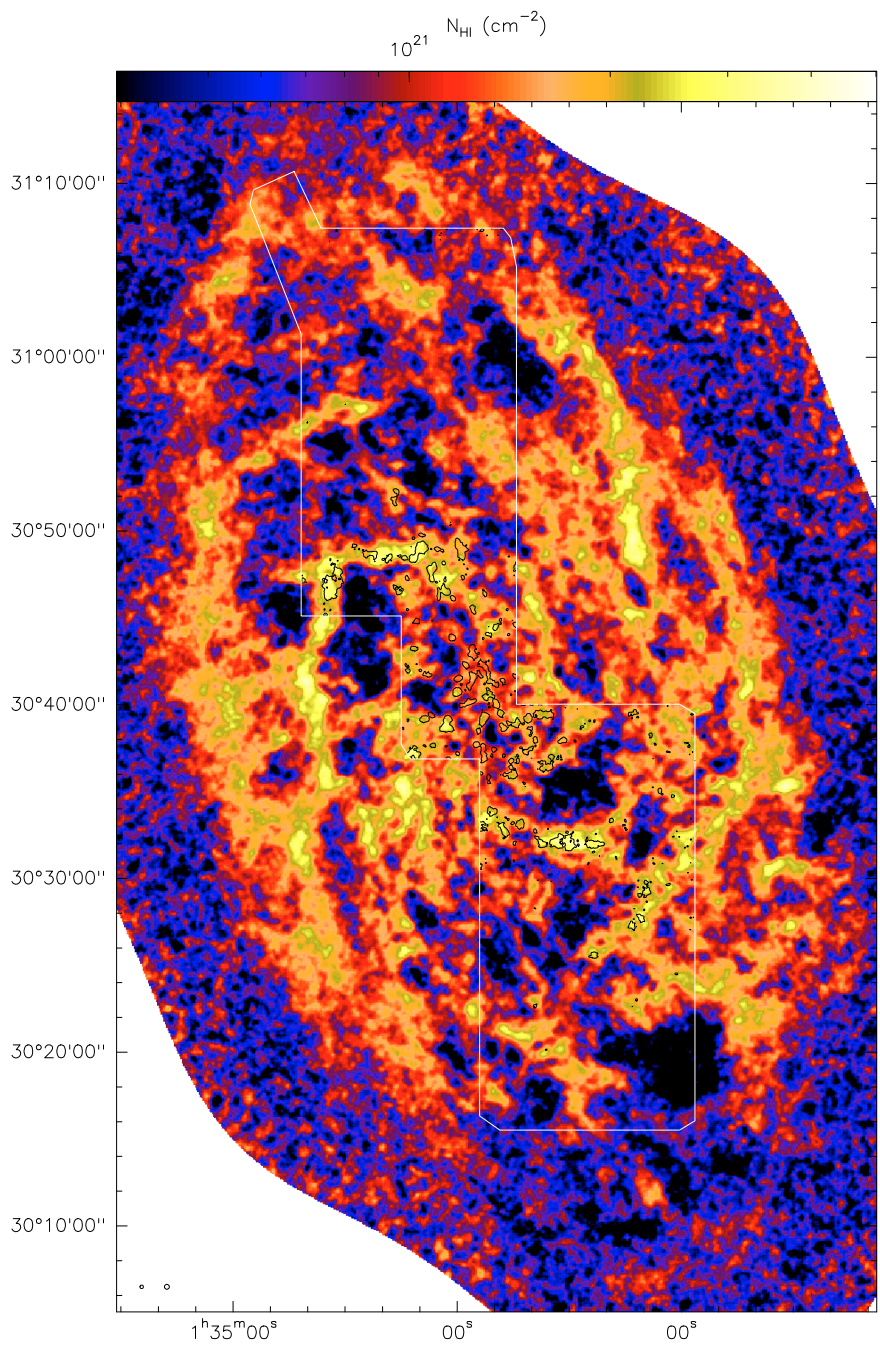

Fig. 5. VLA HI column density map (in color) of the galaxy M 33 at a resolution of $17^{\prime \prime}$ overlaid with the IRAM CO(2-1) intensity contour at $1 \mathrm{~K} \mathrm{~km} \mathrm{~s}^{-1}$. The region observed in $\mathrm{CO}(2-1)$ are marked with thin white lines.

Table 3. Beam and rms noise properties of the H I $21 \mathrm{~cm}$ datacubes.

\begin{tabular}{lccc}
\hline \hline $\begin{array}{l}\text { Beam } \\
\left({ }^{\prime \prime} \times{ }^{\prime \prime}\right)\end{array}$ & $\begin{array}{c}\text { PA } \\
\left({ }^{\circ}\right)\end{array}$ & $\begin{array}{c}\sigma_{\mathrm{S}} \\
(\mathrm{mJy} / \mathrm{beam})\end{array}$ & $\begin{array}{c}\sigma_{\mathrm{T}} \\
(\mathrm{K})\end{array}$ \\
\hline $5.5 \times 5.2$ & -95.1 & 1.1 & 24 \\
$12.0 \times 11.6$ & -31.8 & 2.0 & 9 \\
$17.2 \times 17.1$ & -45.8 & 2.5 & 5.4 \\
$25.9 \times 24.2$ & -74.8 & 2.8 & 2.75 \\
\hline
\end{tabular}

The rms noise, in $\mathrm{mJy} /$ beam and brightness temperature, was calculated over an ellipse of galactocentric radius $8.5 \mathrm{kpc}$ after primary beam correction.

negative bowls surrounding regions of extended emission, and the pedestal of low level uncleaned flux in the final residual map. An extensive comparison of multiscale clean and its point source counterpart has been carried out in Rich et al. (2008). A series of four cubes with angular resolutions of 5, 12, 17, and 25 arcseconds and $1.27 \mathrm{~km} \mathrm{~s}^{-1}$ channel width were computed. The $5^{\prime \prime}$ cube corresponds to the highest resolution achievable with the B array. The other cubes were obtained using Gaussian tapers in the $u v$-plane of respectively 4900 and 3150 and 1900 meters $F W H M$. In order to minimize sidelobes over the entire 
Table 4. Comparison of results with previous studies of M 33.

\begin{tabular}{lrrrr}
\hline \hline CO surveys & $\begin{array}{r}\text { Molecular gas mass } \\
\left(M_{\odot}\right)\end{array}$ & $\begin{array}{r}S F R^{g} \\
\left(M_{\odot} \mathrm{yr}^{-1}\right)\end{array}$ & $\begin{array}{r}\text { Depletion time } \\
\left(10^{8} \mathrm{yr}\right)\end{array}$ & $\begin{array}{r}\text { Depletion time }^{l} \\
\left(10^{8} \mathrm{yr}\right)\end{array}$ \\
\hline This study & $1.9 \times 10^{8}\left(3.3 \times 10^{8}\right)^{c}$ & $0.27^{h}$ & 7 & 7 \\
Gardan et al. (2007) & $3.2 \times 10^{7 d}$ & $0.05-0.13^{i}$ & $2-6$ & $1-3$ \\
Rosolowsky et al. (2007) & $3.6 \times 10^{8 d}$ & $0.45^{j}$ & 8 & $\ldots$ \\
Heyer et al. (2004) & $3.5 \times 10^{8 e}$ & $0.45^{j}$ & 8 & $1-3$ \\
Engargiola et al. (2003) & $9 \times 10^{7 d}$ & $0.24^{i}$ & 4 & $\ldots$ \\
Corbelli (2003) $)^{a}$ & $2.9 \times 10^{8 f}$ & $0.45^{j}$ & 6 & \\
\hline HI surveys & Atomic gas mass & & \\
\hline This study & $1.4 \times 10^{9}$ & & \\
Putman et al. (2009) & $1.5 \times 10^{9 n}$ & & \\
Corbelli \& Schneider (1997) & $2.6 \times 10^{90}$ & & \\
Deul \& van der Hulst (1987) & $1.1 \times 10^{9}$ & & \\
\hline
\end{tabular}

Notes. ${ }^{(a)}$ FCRAO CO(1-0) data from Heyer et al. (2004). ${ }^{(b)}$ All values converted to our adopted $X_{\mathrm{CO}}=4 \times 10^{20} \mathrm{~cm}^{-2} /\left(\mathrm{K} \mathrm{km} \mathrm{s}^{-1}\right)($ twice Galactic). ${ }^{(c)}$ The value in parentheses is the extrapolation to the whole galaxy for comparison with other surveys. See Sect. 3.1.1 for details. (d) Original value used $X_{\mathrm{CO}}=2 \times 10^{20} \mathrm{~cm}^{-2} /\left(\mathrm{K} \mathrm{km} \mathrm{s}^{-1}\right)$ (Galactic). ${ }^{(e)}$ Original value used $X_{\mathrm{CO}}=3 \times 10^{20} \mathrm{~cm}^{-2} /\left(\mathrm{K} \mathrm{km} \mathrm{s}^{-1}\right)$. ${ }^{(f)}$ Original value used $X_{\mathrm{CO}}=2.8 \times 10^{20} \mathrm{~cm}^{-2} /\left(\mathrm{K} \mathrm{km} \mathrm{s}^{-1}\right)$. ${ }^{(g)}$ Over the areas mapped in CO for each survey. ${ }^{(h)}$ From Verley et al. (2009). ${ }^{(i)}$ From $\mathrm{H}_{\alpha}$ with a Kennicutt (1998) calibration. ${ }^{(j)}$ We give the value from Engargiola et al. (2003) as the CO mapped areas are identical. ${ }^{(k)}$ Value computed by dividing the first by the second column of this table. ${ }^{(l)}$ Original value given by the cited papers. ${ }^{(m)}$ All values converted to our adopted distance $D=840 \mathrm{kpc}$. ${ }^{(n)}$ Original value used a distance of $730 \mathrm{kpc}$. Inside a $3.2 \times 10^{20} \mathrm{~cm}^{-2}$ contour. ${ }^{(o)}$ Original value used a distance of $690 \mathrm{kpc}$. Over their whole mapped area extending beyond $8.5 \mathrm{kpc}$.

map, a robust weighting scheme Briggs (1995) was applied to the data using a robustness factor of 0.5 . The rms noise for the cubes was computed over the channels without emission from M 33 within a galactocentric radius of $8.5 \mathrm{kpc}$. Table 3 summarizes the beam sizes and noise properties of the different cubes. A zeroth moment map (Fig. 5 with the CO $1 \mathrm{~K} \mathrm{~km} \mathrm{~s}^{-1}$ intensity contour superposed) was computed at $17^{\prime \prime}$ resolution by masking out regions of the $17^{\prime \prime}$ cube where emission from the $25^{\prime \prime}$ cube was below $2.75 \mathrm{~K}(1 \sigma)$. The data presented here will be made available throught the Centre de Données de Strasbourg (CDS).

\section{Results}

\subsection{Gas masses}

In this section we present measurements of total integrated flux and derived total molecular and atomic gas masses.

\subsubsection{Molecular Gas}

The $\mathrm{CO}(2-1)$ luminosity measured over our observed area is $1.7 \pm 0.1 \times 10^{7} \mathrm{~K} \mathrm{~km} \mathrm{~s}^{-1} \mathrm{pc}^{2}$. Our CO mapped region covers $28 \%$ of the total galaxy area up to $8.5 \mathrm{kpc}$ but $61 \pm 2 \%$ of the infrared emission (see details in Sect. 3.3). The estimated CO(2-1) luminosity for the whole $R_{25}$ disk is thus $2.8 \pm 0.3 \times 10^{7} \mathrm{~K} \mathrm{~km} \mathrm{~s}^{-1} \mathrm{pc}^{2}$. The molecular gas mass is computed with the following hypotheses. Given that metallicity is half solar and the gradient in M 33 is weak $\left(12+\log (\mathrm{O} / \mathrm{H})=8.4-0.03 R_{\mathrm{kpc}}\right.$, Rosolowsky \& Simon 2008; Magrini et al. 2009), we use a constant "standard" Milky Way factor (Dickman et al. 1986) multiplied by a factor of two $N\left(\mathrm{H}_{2}\right) / I_{\mathrm{CO}(1-0)}=4 \times 10^{20} \mathrm{~cm}^{-2} /\left(\mathrm{K} \mathrm{km} \mathrm{s}^{-1}\right)$, implicitly assuming an inverse linear dependence between the conversion factor and metallicity (Wilson 1995) but see Israel (2000). Using the $\mathrm{CO}(1-0)$ observations from Rosolowsky et al. (2007) and our $\mathrm{CO}(2-1)$ observations we compute a $\mathrm{CO}(2-1) / \mathrm{CO}(1-0)$ ratio of 0.8 in the central kiloparsec of M 33. Due to the decrease in the excitation temperature with radius we can expect lower values of the line ratio for the outer parts as found by Sawada et al. (2001) for the Milky Way and Braine et al. (1997) for NGC4414. With a linear gradient of the line ratio from 0.8 at the center of the galaxy to 0.5 at $8.5 \mathrm{kpc}$, and taking into account the measured exponential decrease in the $\mathrm{CO}$ emission with radius (see Sect. 3.3) we find an average $\mathrm{CO}(2-1) /(1-0)$ line ratio of 0.73 for the whole galaxy. The computed mass includes helium by multiplying by a factor 1.37 . The molecular gas mass for the whole galaxy is thus $3.3 \times 10^{8} M_{\odot}$.

\subsubsection{Atomic gas}

The integrated $\mathrm{H}$ I flux density over a region corresponding to a galactocentric radius smaller than $8.5 \mathrm{kpc}$, slightly over the $R_{25}$ radius for $\mathrm{M} \mathrm{33}$, is $8.4 \times 10^{3} \mathrm{Jy} \mathrm{km} \mathrm{s}^{-1}$. The atomic gas mass was derived from the $\mathrm{H}$ I integrated intensity using a conversion factor $1.8 \times 10^{18} \mathrm{~cm}^{-2} /\left(\mathrm{K} \mathrm{km} \mathrm{s}^{-1}\right)$ (Rohlfs \& Wilson 1996). The total $\mathrm{H}$ I mass over the same region is $1.4 \times 10^{9} M_{\odot}$. Putman et al. (2009) using the Arecibo telescope find give $1.5 \times 10^{9} M_{\odot}$ inside a very similar contour which they define as the star forming disk.

\subsection{Kinematics}

We obtain velocity fields for both datasets by computing the first moment of the emission. In the case of $\mathrm{HI}$, the 17" data was masked by the $25^{\prime \prime}$ cube with a threshold of $10 \mathrm{~K}$. For the $\mathrm{CO}$ data, we computed the first moment of the original non H I masked datacube, masking out emission below $4 \sigma$. Both methods are thus completely independent of each other. Fig. 6 shows the $\mathrm{CO}$ and $\mathrm{H}$ I velocity fields at respectively $12^{\prime \prime}$ and $17^{\prime \prime}$. The excellent agreement between the two velocity fields is a further argument in favor of using the H I masking to obtain CO integrated intensity maps. 


\subsection{Radial distribution}

The radial distribution of properties were derived taking azimuthal averages over elliptical rings with inclinations of $i=56^{\circ}$ and position angle PA $=22.5^{\circ}$ (measured toward East from north). The quantities are therefore deprojected from the sky plane onto the plane of M 33. The radial step is 500 pc which corresponds to about $2^{\prime}$. The bottom part of Fig. 7 shows the cumulative fractions of the area (solid line) and $70 \mu \mathrm{m}$ surface brightness (dashed line) in our CO map (black area in the inset) compared to the whole elliptical region of radius less than $R$ (sum of grey and black areas of the inset). For galactocentric distances below $2 \mathrm{kpc}$ our map samples more than $80 \%$ of the accessible disk at these radii both in area and $70 \mu \mathrm{m}$ flux. The whole CO mapped area corresponds to $28 \%$ of the area up to $8.5 \mathrm{kpc}$ but more than $60 \%$ of the corresponding $70 \mu \mathrm{m}$ flux, this is explained by the rapid decrease in infrared emission with radius. The corresponding values for 24 and $160 \mu \mathrm{m}$ are similar to a few percent at each radius. Table 5 summarizes the source and resolution of the ancillary data used.

\subsubsection{Surface brightness}

The top part of Fig. 7 presents the radial variations in average surface brightness of the CO and infrared data from Spitzer at $24 \mu \mathrm{m}, 70 \mu \mathrm{m}$ and $160 \mu \mathrm{m}$, the solid lines correspond to averages taking into account only our CO mapped area and the dashed lines to the whole elliptical annuli for a given radius. For each tracer, the similarity between these two averages is an indication that the area mapped in $\mathrm{CO}$ is representative of the M 33 disk in its entirety. The 4 datasets have similar exponential decreases with radius i.e. $F_{v}(r) \propto \mathrm{e}^{-r / L}$ with a corresponding scale length $L$. Table 6 summarizes the scale lengths computed by least square fittingÊE for CO and IR data, and Table 7 scalelength values from surveys presented in Table 2 .

\subsubsection{Mass surface densities}

We compute mass surface densities for atomic and molecular gas and study their radial distribution. For the molecular gas mass, we use the same hypotheses as in Sect. 3.1.1 including a constant $\mathrm{CO}(2-1) / \mathrm{CO}(1-0)$ factor. Taking a linearly decreasing value of this factor would modify only slightly the radial distribution, raising the most extreme outer points by a factor 1.5 and lowering the inner ones by a factor 1.1, at the price of added uncertainties that cannot be simply estimated. The top panel of Fig. 8 shows the mass surface density of atomic and molecular gas as a function of radius. The atomic gas surface density is constant at an average value of $6 \pm 2 M_{\odot} \mathrm{pc}^{-2}$, the molecular gas mass surface density follows the same trend as the $\mathrm{CO}$ surface brightness presented in Fig. 7. The fraction of molecular to atomic gas in mass is shown in the bottom panel of Fig. 8. Given the constant atomic gas surface density, the fraction decreases following the molecular gas density from 1.2 to 0.015 from the center to the outer parts of the stellar disk.

\subsubsection{Star formation efficiency}

The star formation efficiency is usually defined as the ratio of the star formation rate over the mass of molecular gas available to form stars.

$S F E=S F R / M_{\mathrm{H}_{2}}$.
Table 5. Ancillary data used in this paper.

\begin{tabular}{lcc}
\hline \hline Wavelength & Resolution & Telescope \\
\hline $160 \mu \mathrm{m}$ & $40^{\prime \prime}$ & ${\text { Spitzer } \text { MIPS }^{a}}^{\prime \prime}$ \\
$70 \mu \mathrm{m}$ & $18^{\prime \prime}$ & Spitzer MIPS $^{a}$ \\
$24 \mu \mathrm{m}$ & $6^{\prime \prime}$ & Spitzer MIPS $^{a}$ \\
$8 \mu \mathrm{m}$ & $2^{\prime \prime}$ & Spitzer IRAC $^{b}$ \\
$6570 \AA(\mathrm{H} \alpha)$ & $2^{\prime \prime}$ (pixel size) & KPNO $^{c}$ \\
$1500 \AA$ & $5^{\prime \prime}$ & GALEX FUV $^{d}$ \\
\hline
\end{tabular}

Notes. ${ }^{(a)}$ Hinz et al. (2004); Tabatabaei et al. (2007b). ${ }^{(b)}$ Verley et al. (2007). ${ }^{(c)}$ Hoopes et al. (2001); Greenawalt (1998). ${ }^{(d)}$ Thilker et al. (2005), retrieved from GR5 public release of the MAST archive.

Defined in this way, the SFE is the inverse of the molecular gas depletion time. Estimates from a variety of observations (Blitz \& Rosolowsky 2006; Kennicutt 1998; Hippelein et al. 2003) give a value of the SFR which varies from 0.3 to $0.7 M_{\odot} \mathrm{yr}^{-1}$ for the entire disk. A recent study by Verley et al. (2009) using extinction corrected $\mathrm{H} \alpha, \mathrm{FUV}$, and infrared tracers gives a value of $0.45 \pm 0.1 M_{\odot} \mathrm{yr}^{-1}$ for total SFR. This is our adopted value.

Our CO map does not cover the whole disk but covers respectively $63 \%, 61 \%$ and $59 \%$ of the IR emission at $24 \mu \mathrm{m}$, $70 \mu \mathrm{m}$ and $160 \mu \mathrm{m}$ (see bottom panel of Fig. 7) so the $S F R$ is $\sim 0.27 M_{\odot} \mathrm{yr}^{-1}$ within the area covered by our CO map. With a $N\left(\mathrm{H}_{2}\right) / I_{\mathrm{CO}(1-0)}$ value of $4 \times 10^{20} \mathrm{~cm}^{-2} /\left(\mathrm{K} \mathrm{km} \mathrm{s}^{-1}\right)$ and an average $2-1 / 1-0$ line ratio of 0.73 (see Sect. 3.1.1 for details), the molecular gas mass within the CO map is $1.9 \times 10^{8} \mathrm{M}_{\odot}$. With these values, the SFE is $1.4 \times 10^{-9} \mathrm{yr}^{-1}$ or a molecular gas depletion time $7 \times 10^{8} \mathrm{yr}$. An SFE of $1.6 \times 10^{-9} \mathrm{yr}^{-1}$ is a factor $\sim 3$ higher than that found in large spiral galaxies (sample in, Kennicutt 1998; Murgia et al. 2002; Leroy et al. 2008) Earlier studies used lower $N\left(\mathrm{H}_{2}\right) / I_{\mathrm{CO}(1-0)}$ values and thus obtained even higher SFEs (or shorter $\mathrm{H}_{2}$ depletion times) as is shown in Table 4.

In order to bring the SFE of M 33 to the same level as in large spirals, the $N\left(\mathrm{H}_{2}\right) / I_{\mathrm{CO}(1-0)}$ factor would have to be about 3 times the value we use whereas previous studies used even smaller values (Wilson \& Scoville 1990; Corbelli 2003; Heyer et al. 2004; Engargiola et al. 2003; Rosolowsky et al. 2007; Gardan et al. 2007). Through Virial mass estimates and ${ }^{13 / 12} \mathrm{CO}$ line ratios, Braine et al. (2010) support a value of about $N\left(\mathrm{H}_{2}\right) / I_{\mathrm{CO}(1-0)}=$ $4 \times 10^{20} \mathrm{~cm}^{-2} /\left(\mathrm{K} \mathrm{km} \mathrm{s}^{-1}\right)$ in the disk of M 33, possibly closer to the Galactic value in the inner disk.

Figure 9 presents the radial distributions of the SFE as calculated from the $\mathrm{H} \alpha, 24$, and $70 \mu \mathrm{m}$ emission. The units are arbitrary as we are interested in showing the radial trend. The SFR were taken as being directly proportional to the $\mathrm{H} \alpha$ and IR luminosities. $\mathrm{H} \alpha$ and FIR emission tend to show opposite biases - the $\mathrm{H} \alpha$ suffers extinction where the FIR is strong and the FIR is weak where little dust is present but the $\mathrm{H} \alpha$ is unaffected. If one were to compute the SFE taking into account the total gas mass, the SFE would drop dramatically with increasing radius as opposed to the SFE computed using only molecular gas that does not appear to vary with radius. Table 4 summarizes the masses, star formation rates and depletion times for this study and previous surveys of M 33.

The infrared data scale lengths agree with the values in Tabatabaei et al. (2007a) and Verley et al. (2009), although they are slightly smaller because the IR tracers seem to marginally drop more rapidly in our $\mathrm{CO}$ mapped region compared to the 


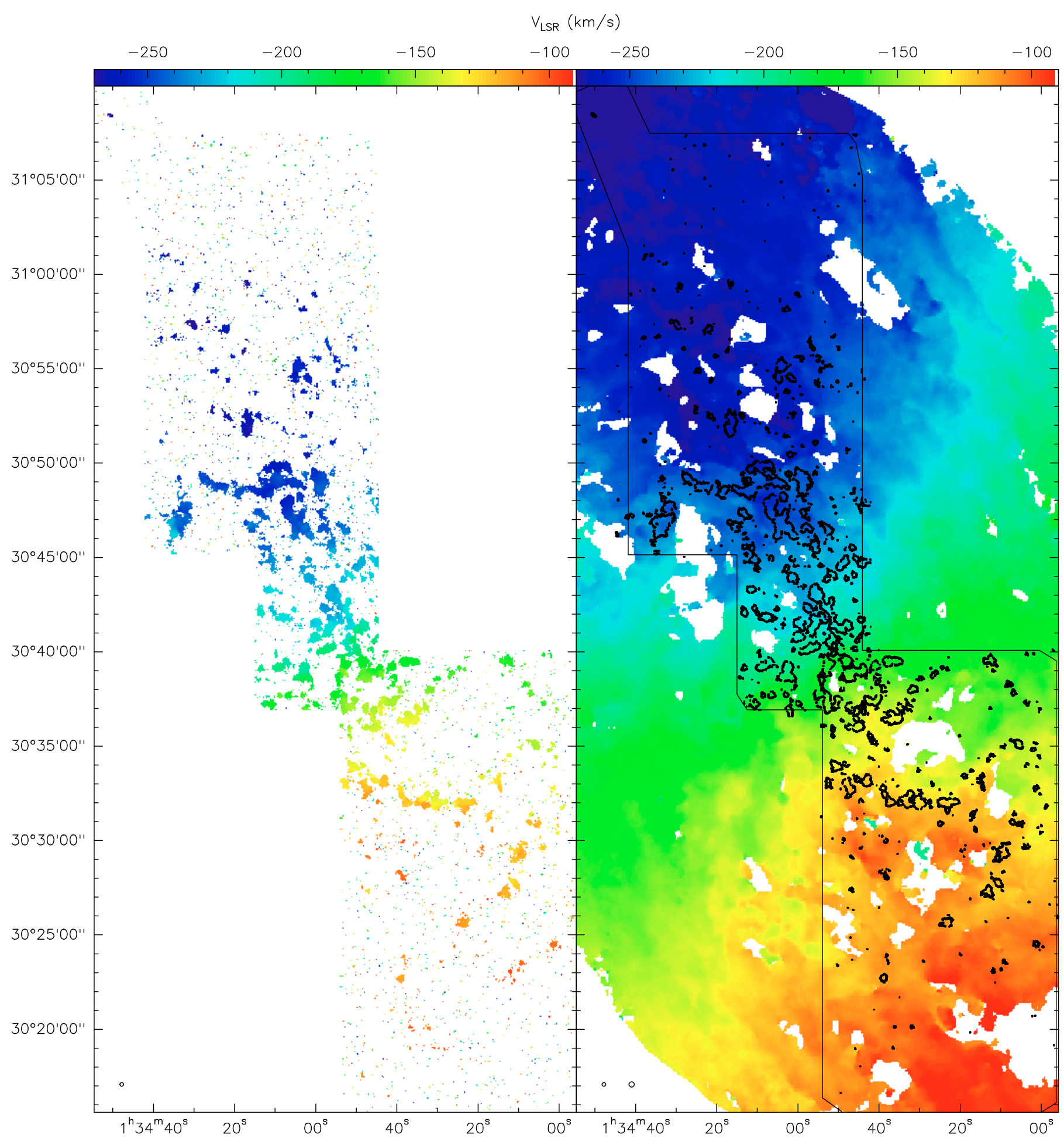

Fig. 6. $\mathrm{CO}($ left $)$ and $\mathrm{HI}$ (right) velocity fields at respectively $12^{\prime \prime}$ and $17^{\prime \prime}$. The $\mathrm{CO}$ velocity field was computed from the original non $\mathrm{H}$ I masked cube, both velocity fields are thus independent. The contours on the right correspond to a constant $\mathrm{S} / \mathrm{N}$ ratio of 10 in the $12^{\prime \prime} \mathrm{CO}$ integrated intensity.

whole galaxy. The scale length of $\mathrm{CO}$ is higher than the ones of $24 \mu \mathrm{m}$ and $70 \mu \mathrm{m}$ and similar to the $160 \mu \mathrm{m}$.

\subsection{The ISM and star formation tracers}

In this section, we compare the $\mathrm{CO}$ emission with tracers of star formation. We present a series of figures (10 to 14) comparing the $\mathrm{CO}$ emission, as contours, overlaid on images of the 8,24 and $70 \mu \mathrm{m}$ FIR emission as well as the $\mathrm{H} \alpha$ and FUV emission (Blow ups of these 5 figures are available in the online version of this article). The $160 \mu \mathrm{m}$ image is at lower resolution and brings little morphological information not present in the $70 \mu \mathrm{m}$ image.

While the dynamic range in the $\mathrm{CO}$ image is much lower than in the images of star formation tracers, the correspondence 
P. Gratier et al.: Molecular and atomic gas in the Local Group galaxy M 33
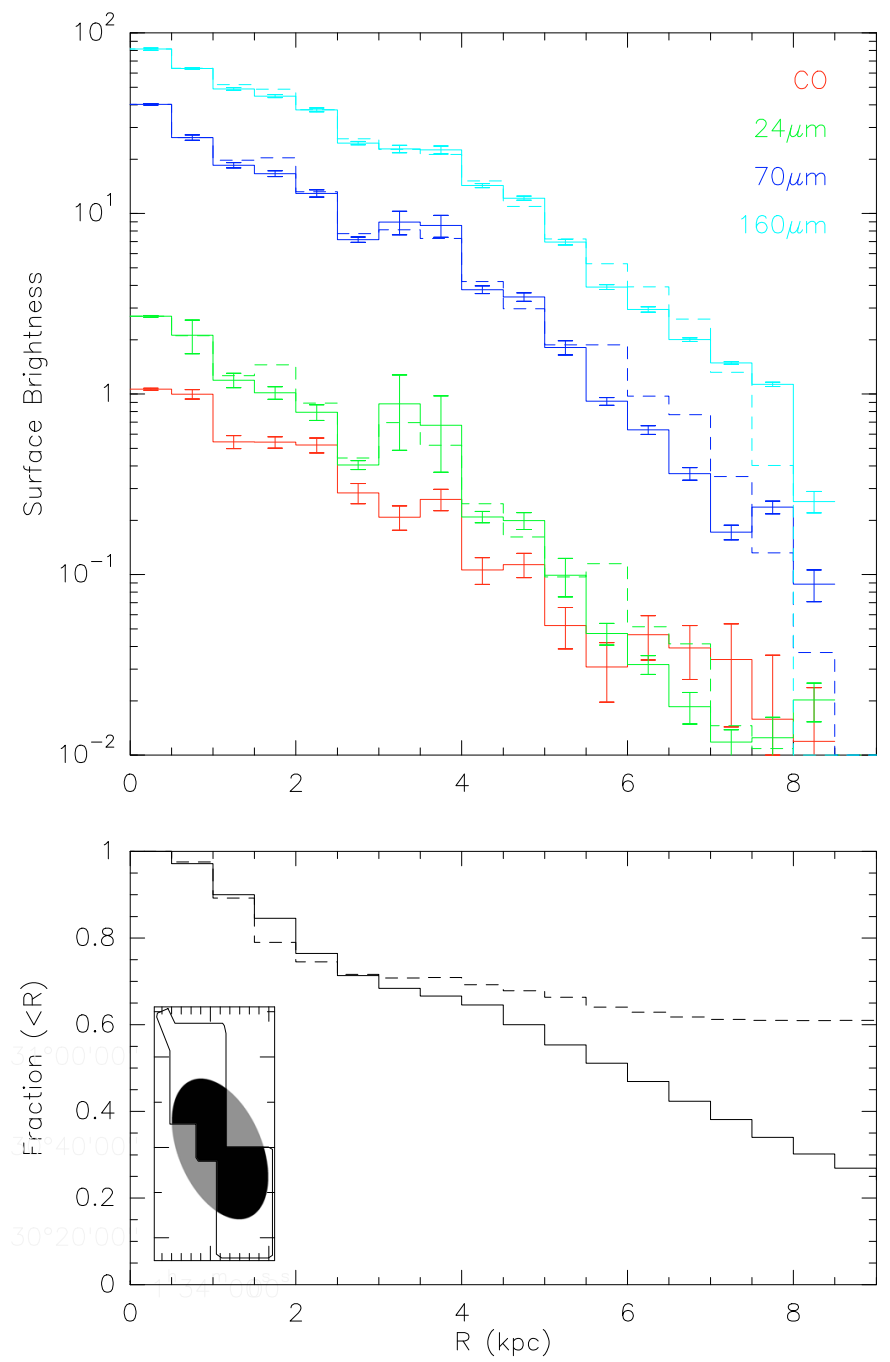

Fig. 7. (Color online) Top: radial distribution of the CO and IR surface brightness in units of MJy sr${ }^{-1}$ for infrared data and $\mathrm{K} \mathrm{km} \mathrm{s}^{-1}$ for $\mathrm{CO}$. For the dust tracers, the surface brightness is computed both on the region covered by our $\mathrm{CO}$ observations (solid line) and on the whole elliptic annuli for each radius (dashed lines), showing that our CO sampled area is representative of the galaxy for each radius. Bottom: cumulative fractions of the area (solid line) and $70 \mu \mathrm{m}$ surface brightness (dashed line) in the region covered by our $\mathrm{CO}$ observations (black area in the lower left diagram) compared to the whole elliptic region of radius less than $R$ (sum of grey and black areas at a given $R$ ).

Table 6. Exponential scale length $L$ in kpc for Spitzer MIPS and CO emission computed over the area mapped in CO.

\begin{tabular}{lrrr}
\hline \hline & {$[0.5-3.5] \mathrm{kpc}$} & {$[3.5-7] \mathrm{kpc}$} & {$[0.5-7] \mathrm{kpc}$} \\
\hline $24 \mu \mathrm{m}$ & $1.36 \pm 0.05$ & $1.02 \pm 0.07$ & $1.40 \pm 0.03$ \\
$70 \mu \mathrm{m}$ & $1.51 \pm 0.07$ & $1.05 \pm 0.06$ & $1.48 \pm 0.04$ \\
$160 \mu \mathrm{m}$ & $2.26 \pm 0.12$ & $1.30 \pm 0.06$ & $1.83 \pm 0.07$ \\
$\mathrm{CO}$ & $2.0 \pm 0.2$ & $1.6 \pm 0.3$ & $1.9 \pm 0.1$ \\
\hline
\end{tabular}

is globally excellent. Figure 16 shows the scatter plot between the $\mathrm{CO}$ integrated intensity and $70 \mu \mathrm{m}$ emission.

Figures 10-14 show that in almost all cases the CO emission is found where star formation is detected and in general the $\mathrm{CO}$ emission is stronger as the level of SF increases. The weaker $\mathrm{CO}$ contours outline the regions with a remarkable precision (see
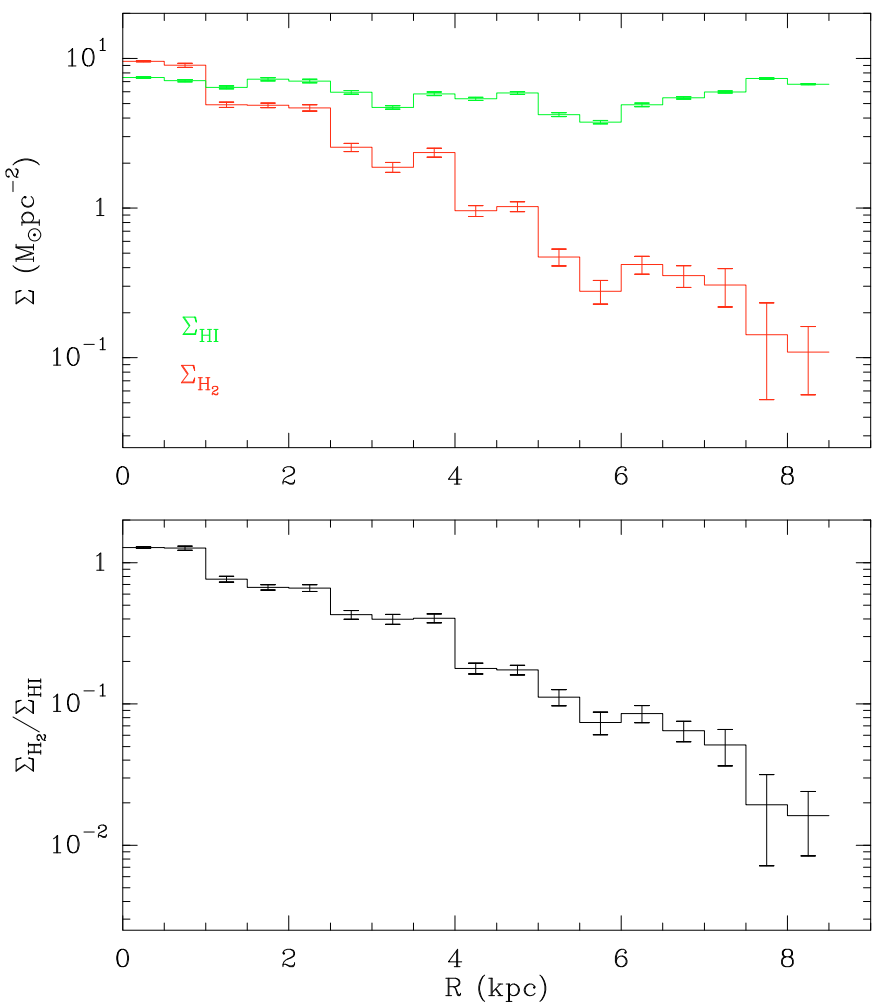

Fig. 8. Top: radial distribution of the $\mathrm{H}_{2}$ and $\mathrm{H}$ I mass surface density. Bottom: radial distribution of the molecular to atomic mass fraction.

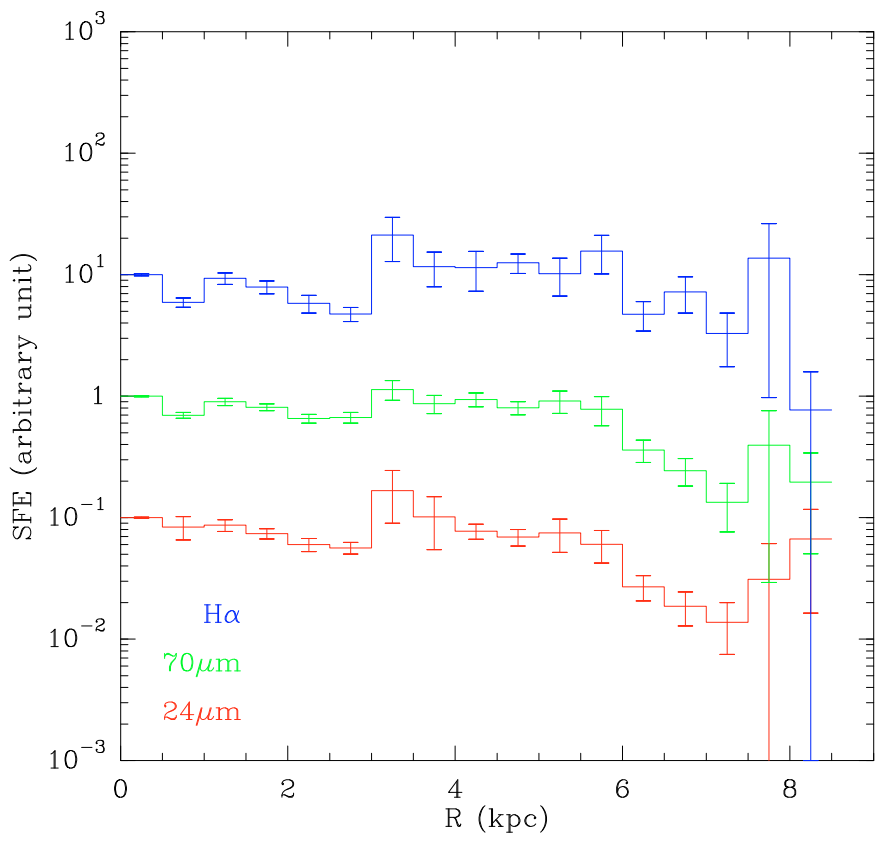

Fig. 9. Radial distribution of the SFE in arbitrary units, all of the curves are normalized to the central value and shifted by 1 decade along the vertical axis.

online Figs. 1 to 12 for the blowups). A partial exception is found near $\left(\alpha=1^{\mathrm{h}} 34^{\mathrm{m}} 09^{\mathrm{s}} .4, \delta=+30^{\circ} 49^{\prime} 06^{\prime \prime}, \mathrm{J} 2000\right)$ where the CO emission is extremely strong while the SF tracers show rather weak emission. The $\mathrm{CO}$ spectrum in the direction of that particular cloud is shown in Fig. 17.

A true exception to the FIR-HI-CO correlation is the interarm cloud at $\left(\alpha=1^{\mathrm{h}} 34^{\mathrm{m}} 16^{\mathrm{s}} .7, \delta=+30^{\circ} 59^{\prime} 3^{\prime \prime}, \mathrm{J} 2000\right)$ dubbed 


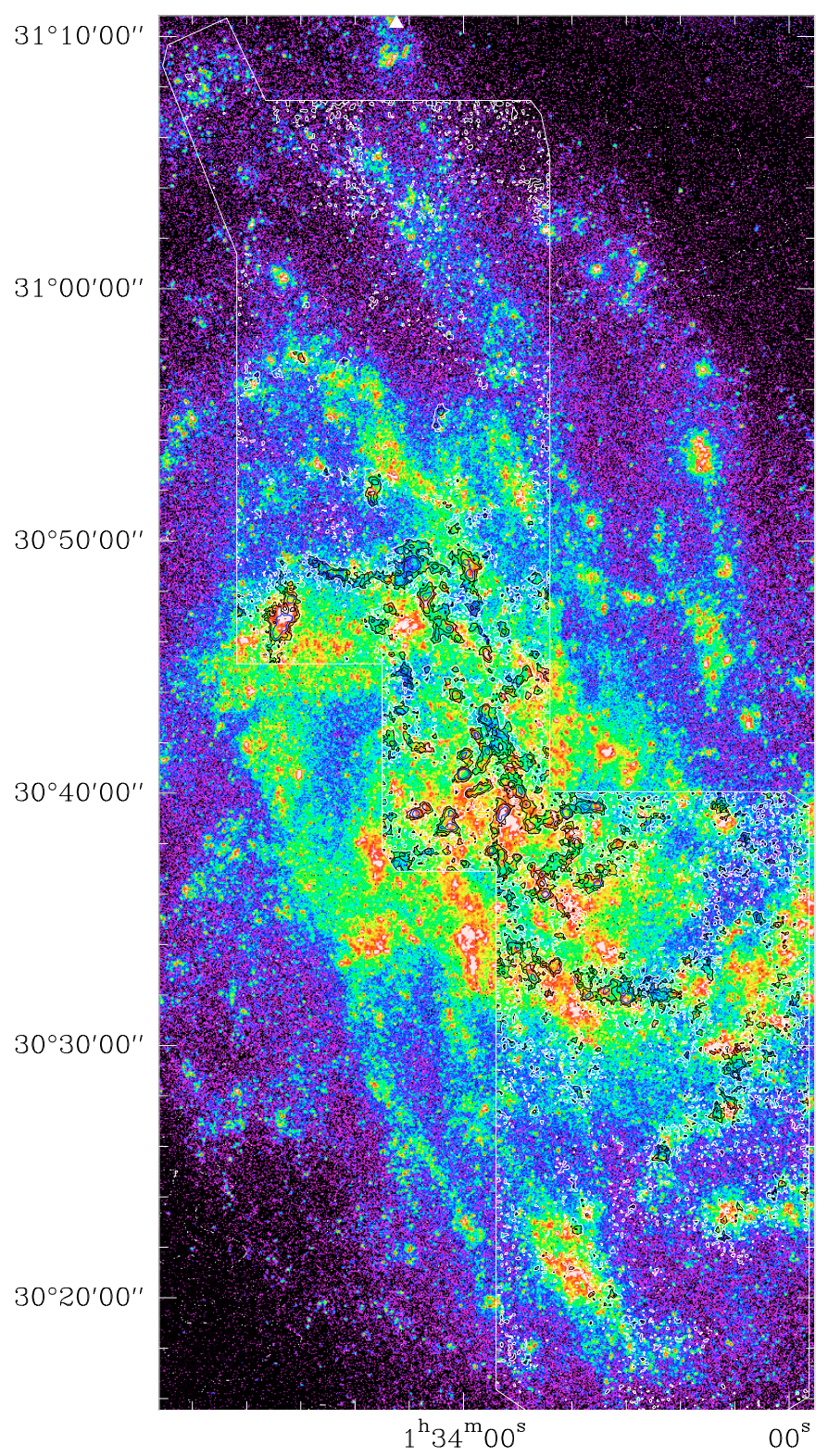

Fig. 10. Galex FUV image with $I_{\mathrm{CO}}$ main beam contours of 1 (white), 2, 4 (black), 8 (blue) $\mathrm{K} \mathrm{km} \mathrm{s}^{-1}$. The beam size is shown as a white dot in the lower left corner. Blow ups are available in the online version.

Table 7. Comparison with previous surveys of $\mathrm{CO}$ emission exponential scale lengths $L$ in kpc.

\begin{tabular}{lcc}
\hline \hline Study & $\begin{array}{c}\text { Range } \\
(\mathrm{kpc})\end{array}$ & $\begin{array}{c}\text { Scalelength } \\
(\mathrm{kpc})\end{array}$ \\
\hline This paper & $0-7$ & $1.9 \pm 0.1$ \\
Gardan et al. (2007) & $2-6$ & $1.4 \pm 0.1$ \\
Engargiola et al. (2003) & $0-7$ & $1.4 \pm 0.1$ \\
Corbelli (2003) & $0-6$ & 2.5 \\
\hline
\end{tabular}

"Lonely Cloud" by Gardan et al. (2007). It is found in a region with very little if any star formation and relatively weak $\mathrm{H}$ I emission, despite fairly strong $\mathrm{CO}$ (and even ${ }^{13} \mathrm{CO}$ ) emission. When H I brightness thresholds higher than $10 \mathrm{~K}$ are used to create the masked zero-moment CO maps, the Lonely Cloud disappears. While this cloud was discovered in the first set of

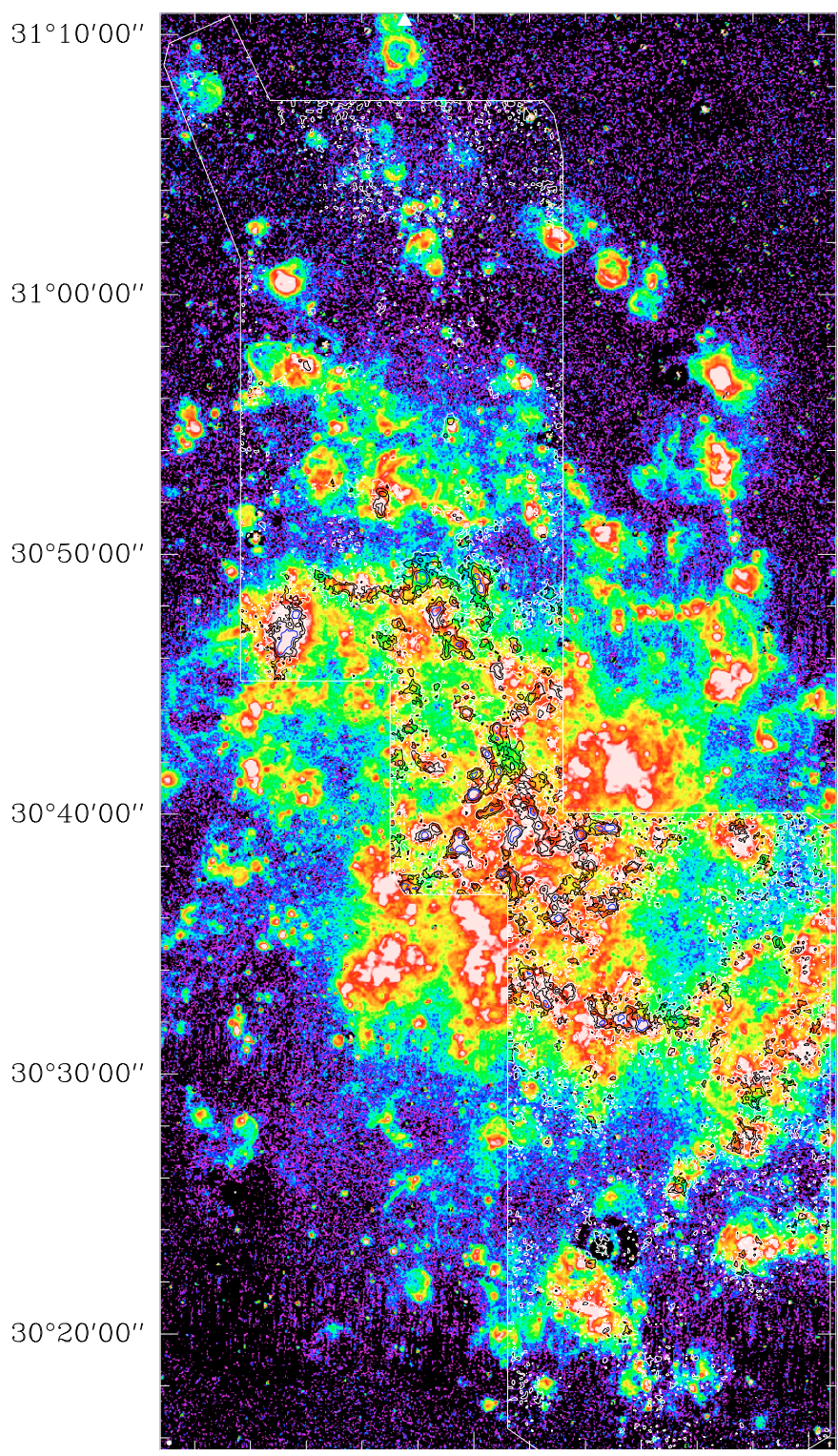

$1^{\mathrm{h}} 34^{\mathrm{m}} 00^{\mathrm{s}}$

$00^{\mathrm{S}}$

Fig. 11. KPNO $\mathrm{H} \alpha$ image with $I_{\mathrm{CO}}$ main beam contours of 1 (white), 2, 4 (black), 8 (blue) $\mathrm{K} \mathrm{km} \mathrm{s}^{-1}$. The beam size is shown as a white dot in the lower left corner. Blow ups are available in the online version.

observations, it remains a unique object as the observations of the central and southern regions, despite considerably lower noise levels than in Gardan et al. (2007), have not revealed similar molecular clouds.

In the outer parts, the FIR emission is typically quite inconspicuous, even where $\mathrm{CO}$ has been detected, but the $\mathrm{H} \alpha$ emission is often quite strong. In the central regions, the alignment of the emission at the different wavelengths is nearly perfect, especially between $\mathrm{CO}$ and FIR. Further out, however, notable offsets become more common although there is a clear association between the $\mathrm{CO}$ and FIR/H $\alpha$ emitting regions. The offsets are less than $100 \mathrm{pc}$, particularly in comparison with the FIR.

The CO emission follows HI-defined arm-like structures very closely, completely avoiding the $\mathrm{H}$ I holes. As can be seen in Fig. 15 and corresponding blow ups in the online version of the paper, the overall correlation between $\mathrm{HI}$ and $\mathrm{CO}$ emission is very good. CO is generally found in HI-rich regions although not 
P. Gratier et al.: Molecular and atomic gas in the Local Group galaxy M 33

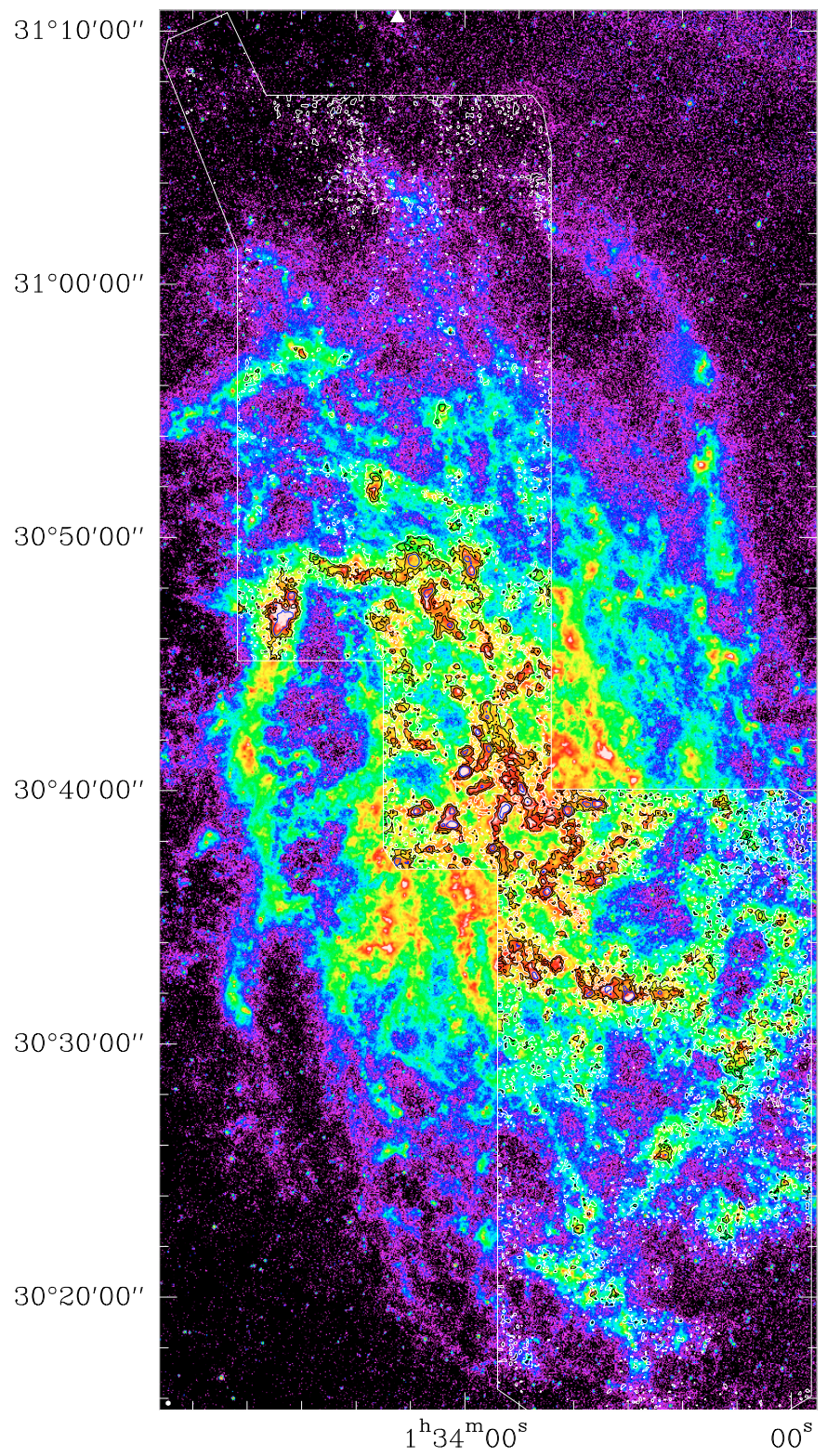

Fig. 12. Spitzer $8 \mu \mathrm{m}$ image with $I_{\mathrm{CO}}$ main beam contours of 1 (white), 2, 4 (black), 8 (blue) $\mathrm{K} \mathrm{km} \mathrm{s}^{-1}$. The beam size is shown as a white dot in the lower left corner. Blow ups are available in the online version.

all of the $\mathrm{H}$ I peaks show $\mathrm{CO}$ emission and not all $\mathrm{CO}$ emission falls on HI. It is not clear what the source of the $70 \mu \mathrm{m}$ emission is in for example the $\mathrm{HI}$ (and CO) hole around $\left(\alpha=1^{\mathrm{h}} 34^{\mathrm{m}} 25\right.$, $\delta=+30^{\circ} 35^{\prime} 00^{\prime \prime}, \mathrm{J} 2000$ ) as the amount of neutral gas is very low. The emission in this region is lower in the 24 and $8 \mu \mathrm{m}$ wavebands, so perhaps the 70 micron emission appears stronger than in reality due to the poorer spatial resolution. The upcoming Herschel HERM33ES observations should answer this.

\subsection{Examples of interesting spectra}

The spectra shown in Figs. 17-19 are just a few among a huge number but are intended to show "special" positions. Figure 17 shows a very strong CO profile towards a region without strong star formation (note good agreement with Engargiola et al. 2003). The line is very strong with a line width of about $11 \mathrm{~km} \mathrm{~s}^{-1}$ at half-intensity. The other spectra shown are

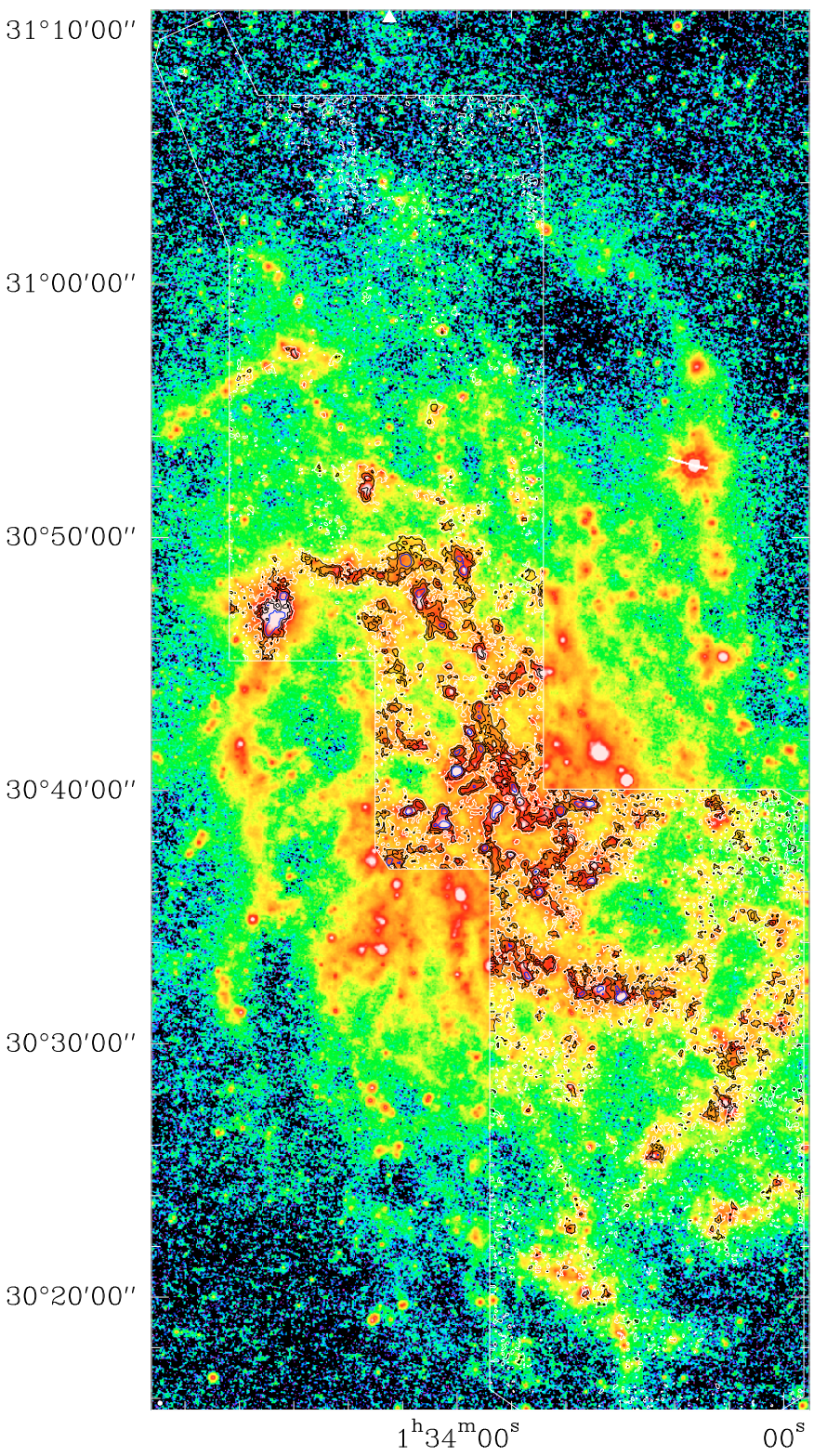

Fig. 13. Spitzer $24 \mu \mathrm{m}$ image with $I_{\mathrm{CO}}$ main beam contours of 1 (white), 2, 4 (black), 8 (blue) $\mathrm{K} \mathrm{km} \mathrm{s}^{-1}$. The beam size is shown as a white dot in the lower left corner. Blow ups are available in the online version.

interesting because the $\mathrm{CO}$ lines are detected very far from the center of M 33, despite the low-metallicity and surface mass density. Figure 18 shows the second molecular cloud detected at or beyond $R_{25}$ in M 33. A secondary peak offset in velocity by about $8 \mathrm{~km} \mathrm{~s}^{-1}$ may be present. Figure 19 shows further spectra near $R_{25}$ which clearly is composed of emission from two clouds, the cloud to the SE (of the figure) at $-260 \mathrm{~km} \mathrm{~s}^{-1}$ and the one to the $\mathrm{NW}$ at about $-273 \mathrm{~km} \mathrm{~s}^{-1}$. The spectral resolution of the data is barely sufficient to measure the linewidths of the clouds, which are typically less than $5 \mathrm{~km} \mathrm{~s}^{-1}$ at half power. This was also true of the spectra of distant clouds shown in Braine et al. (2010) they are much narrower than in the $\mathrm{H}_{2}$-rich inner disk. It is not clear whether the broad linewidths in the inner disk are because the GMCs themselves are much more massive or due to a superposition of clouds (or a single GMC in a region with significant diffuse emission). 


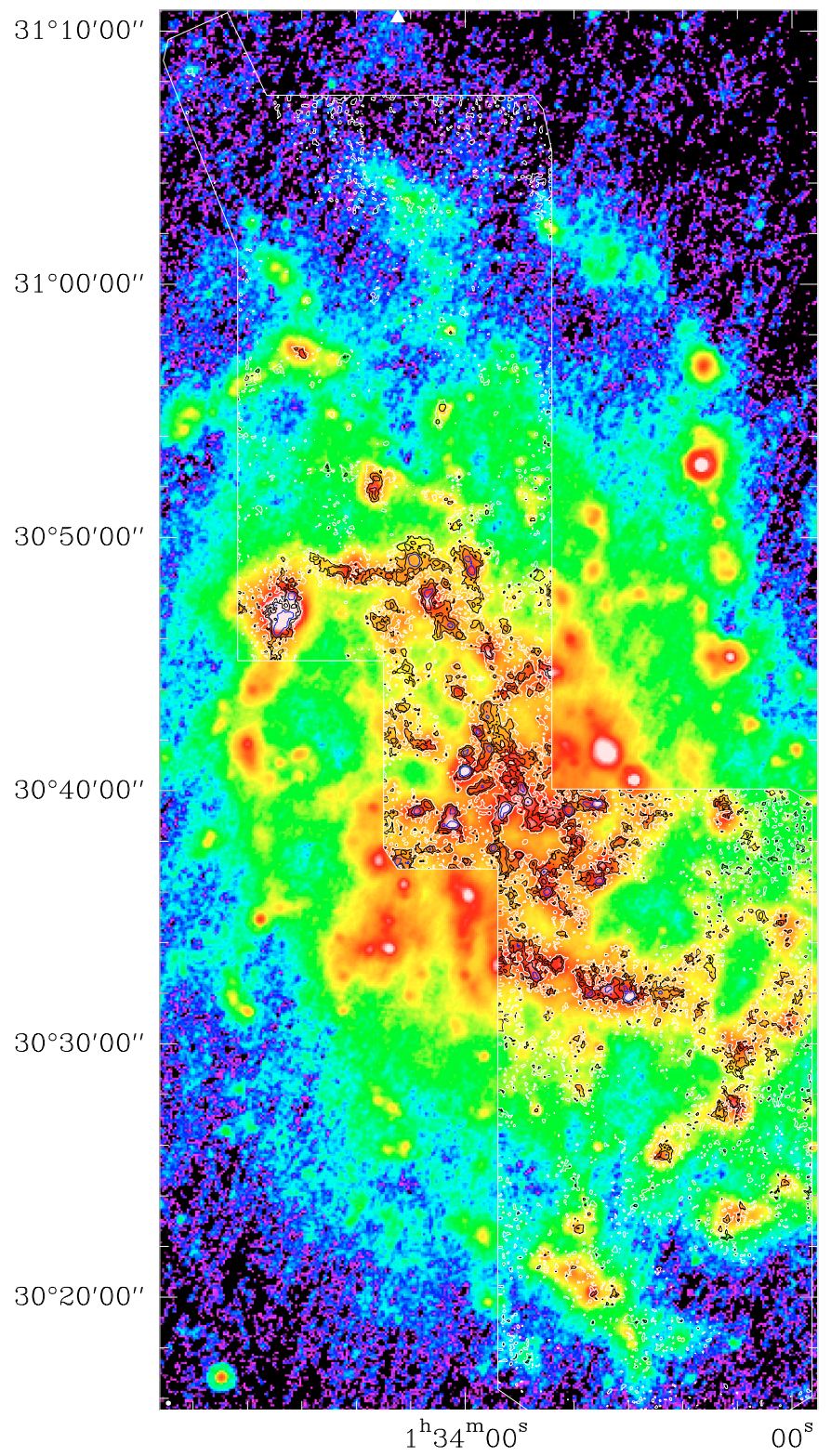

Fig. 14. Spitzer $70 \mu \mathrm{m}$ image with $I_{\mathrm{CO}}$ main beam contours of 1 (white), 2, 4 (black), 8 (blue) $\mathrm{K} \mathrm{km} \mathrm{s}^{-1}$. The beam size is shown as a white dot in the lower left corner. Blow ups are available in the online version.

The spectra shown also illustrate the dynamic range of the data acquired, roughly 100 in integrated intensity.

\section{Conclusions}

This work presents high-resolution maps of the atomic and molecular gas in the disk of M 33 via observations of the $\mathrm{HI} 21 \mathrm{~cm}$ and $\mathrm{CO}(2-1)$ lines. The whole disk out to $8.5 \mathrm{kpc}$ is covered in the $\mathrm{HI}$ line and about $60 \%$ of the emission in CO.

Assuming the $N\left(\mathrm{H}_{2}\right) / I_{\mathrm{CO}(1-0)}$ factor to be twice that of the Galaxy, because of the subsolar metallicity of M 33, we estimate a molecular gas mass of $3.3 \times 10^{8} M_{\odot}$ roughly $20 \%$ of the $1.4 \times 10^{9} M_{\odot}$ detected in the inner $8.5 \mathrm{kpc}$ in H I. Azimuthally averaging, the $\mathrm{HI}$ surface density is close to constant with radius but the $\mathrm{H}_{2}$ decreases exponentially with a scale length of $1.9 \mathrm{kpc}$. The $\mathrm{H}_{2} / \mathrm{HI}$ mass ratio decreases from about unity to $1 \%$.

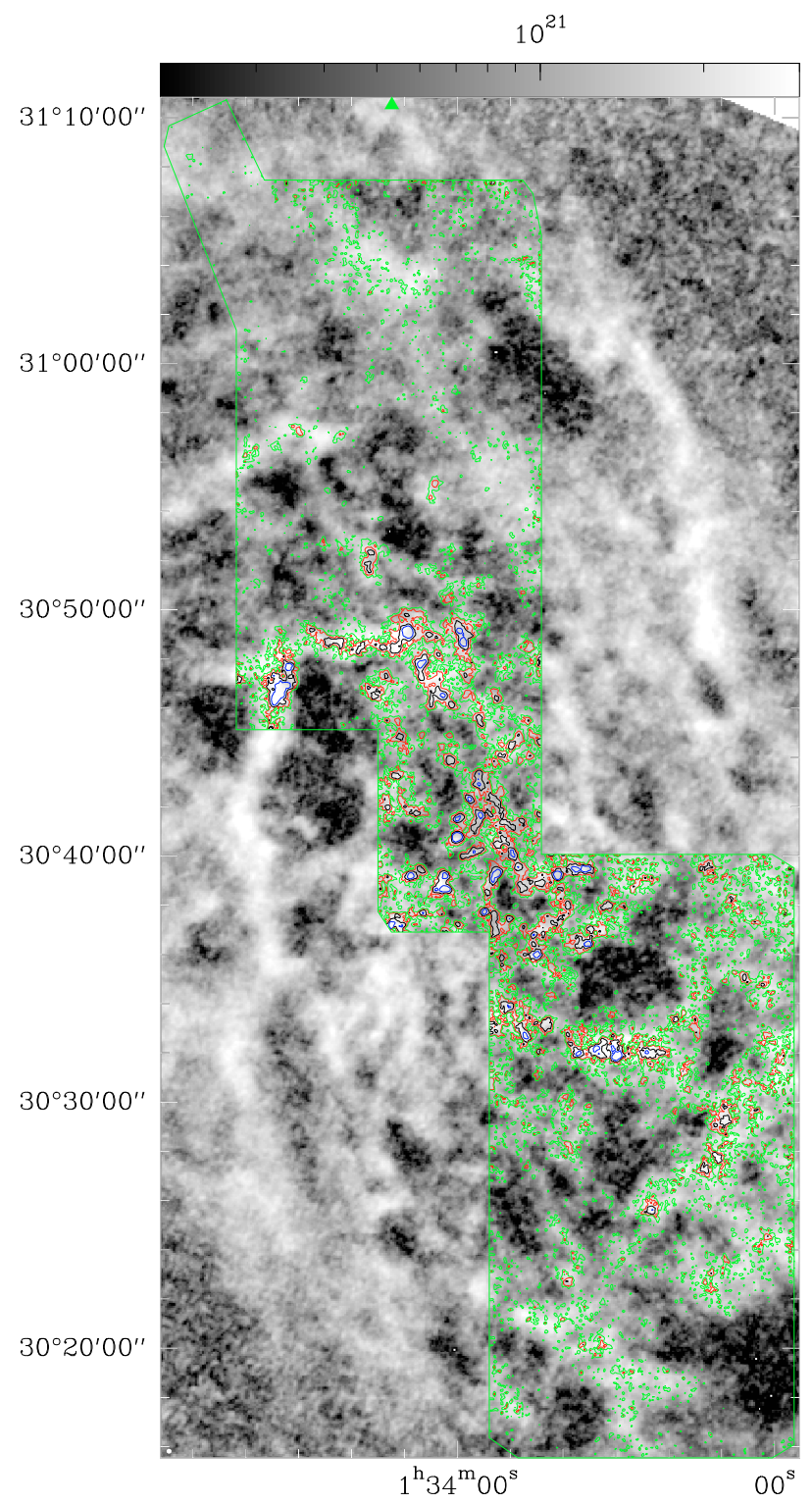

Fig. 15. VLA H I zeroth moment image with $I_{\mathrm{CO}}$ main beam contours of 1 (green), 2 (red), 4(black), 8 (blue) $\mathrm{K} \mathrm{km} \mathrm{s}^{-1}$. The beam size is shown as a white dot in the lower left corner. Note how the $\mathrm{CO}$ emission follows the bright $\mathrm{HI}$ and outlines the $\mathrm{H}$ I holes. Blow ups are available in the online version.

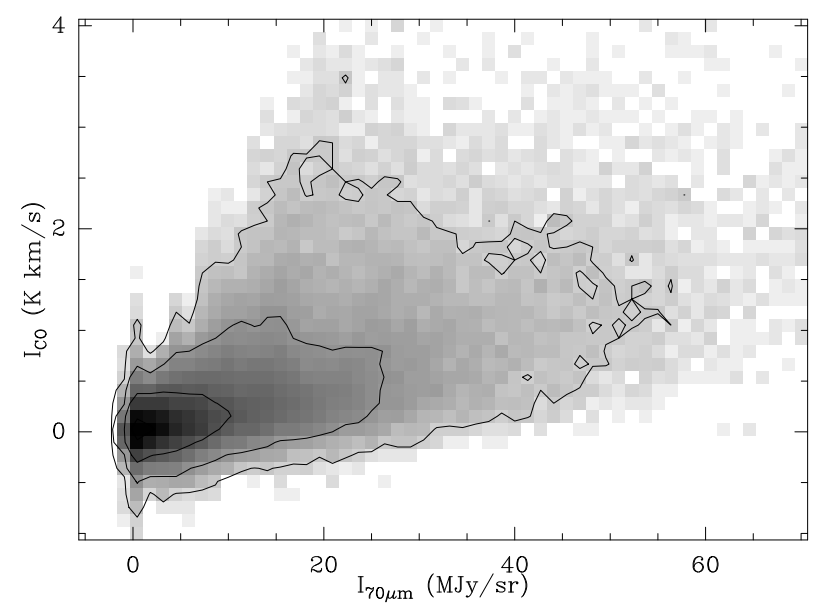

Fig. 16. Scatter plot between $\mathrm{CO}$ integrated intensity and $70 \mu \mathrm{m}$ emission. 
P. Gratier et al.: Molecular and atomic gas in the Local Group galaxy M 33

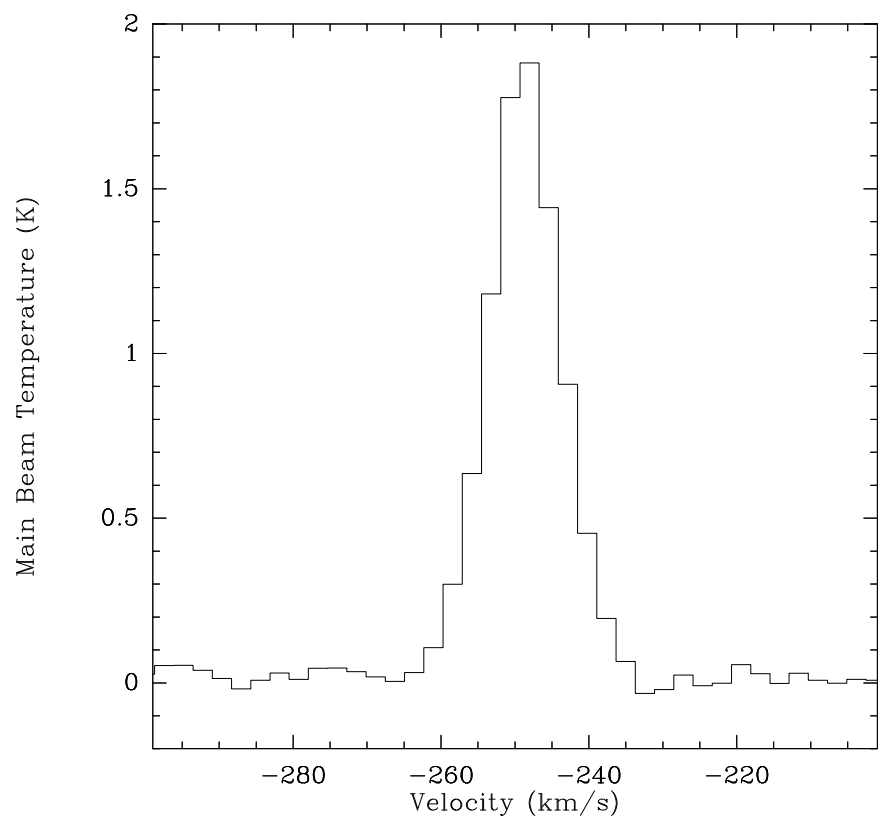

Fig. 17. Spectrum of a bright cloud at position $\left(\alpha=1^{\mathrm{h}} 34^{\mathrm{m}} 09^{\varsigma} 4, \delta=\right.$ $+30^{\circ} 49^{\prime} 06^{\prime \prime}, \mathrm{J} 2000$ ), unlike other strong peak of CO integrated intensity, it lacks strong emission in star formation tracers (see Sect. 3.4).

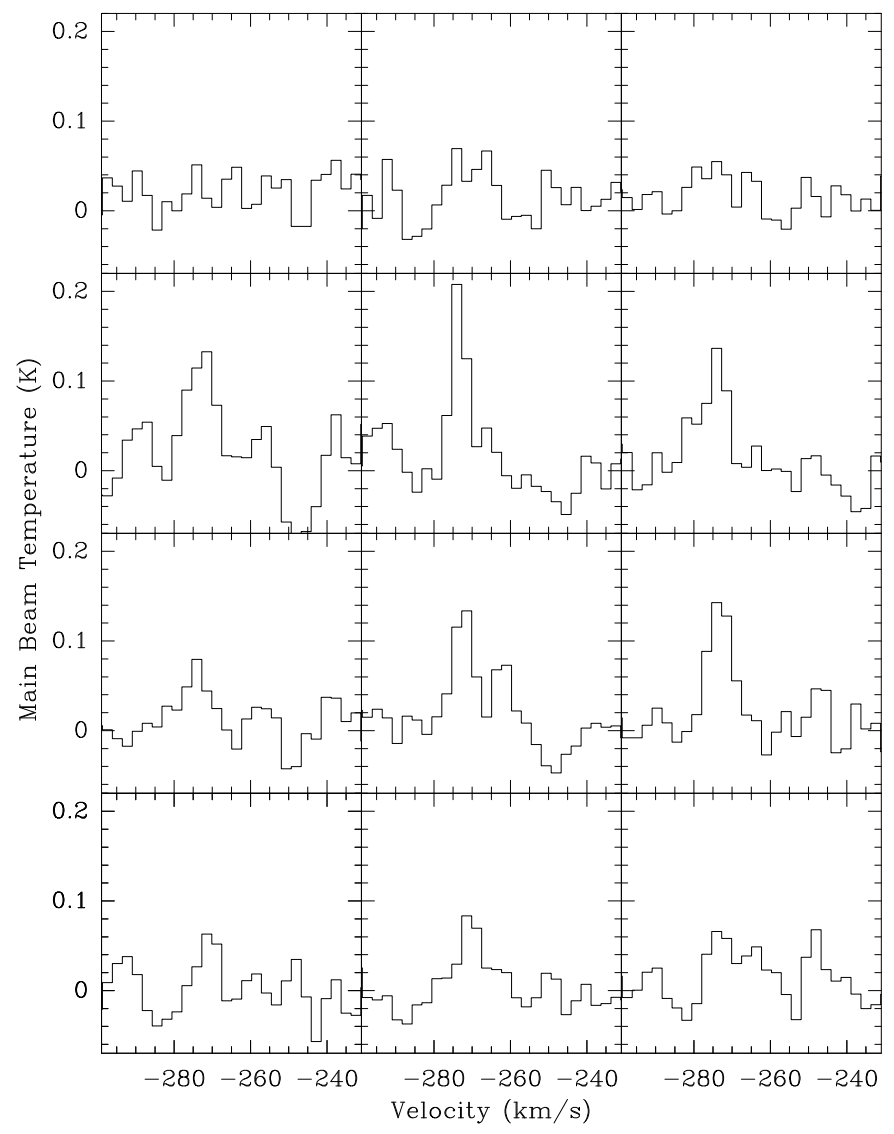

Fig. 18. Spectra of the outer disk cloud $\left(\alpha=1^{\mathrm{h}} 34^{\mathrm{m}} 50^{\mathrm{s}} .8, \delta=\right.$ $+31^{\circ} 08^{\prime} 26^{\prime \prime}, \mathrm{J} 2000$ ) shown with $6^{\prime \prime}$ spacing between panels. If the secondary peak at $-266 \mathrm{~km} \mathrm{~s}^{-1}$, seen in the second spectrum from the bottom in the middle column, is real then the cloud is small as it is only seen within a single beam.

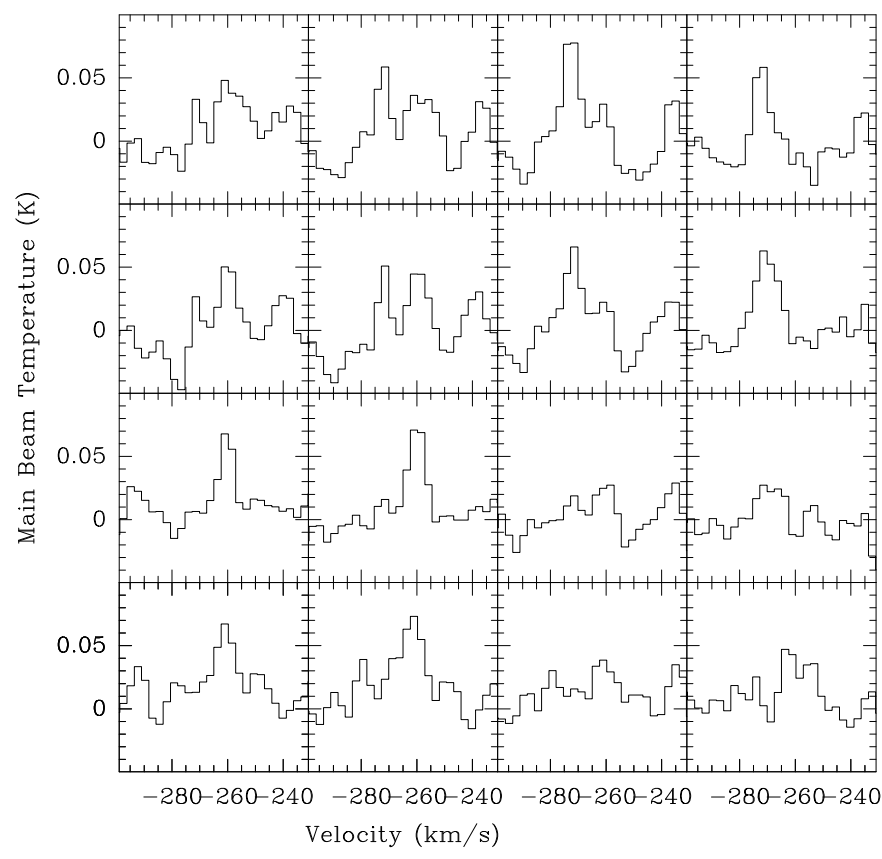

Fig. 19. Spectra of two more outer disk clouds at different velocities. The lower left panel is at $\left(\alpha=1^{\mathrm{h}} 34^{\mathrm{m}} 21^{\mathrm{s}} .7, \delta=+31^{\circ} 04^{\prime} 13^{\prime \prime}, \mathrm{J} 2000\right)$ and the panel spacing is $6^{\prime \prime}$.

The correspondence between the peaks and holes in the distributions of molecular and atomic gas is excellent and follows the peaks and troughs in the FIR, MIR, and $\mathrm{H} \alpha$ images. The SFE is approximately constant with radius, suggesting that molecular gas is transformed into stars at a similar rate (assuming a similar IMF) at all galactocentric radii. However, the SFE in the small, gas-rich, low-metallicity, blue spiral M 33 appears 2-4 times higher than what is observed in large nearby spirals.

The sensitivity of the survey is such that $\mathrm{CO}$ emission is detected far out in the disk of M 33 although few clouds are present and the lines are much weaker in intensity.

Acknowledgements. We thank the IRAM staff in Granada for their help with the observations.

\section{References}

Adler, D. S., Lo, K. Y., Wright, M. C. H., et al. 1992, ApJ, 392, 497 Blitz, L., \& Rosolowsky, E. 2006, ApJ, 650, 933

Braine, J., Brouillet, N., \& Baudry, A. 1997, A\&A, 318, 19

Braine, J., Gratier, P., Kramer, C., et al. 2010, A\&A, 520, A107

Briggs, D. S. 1995, in BAAS, 27, 1444

Corbelli, E. 2003, MNRAS, 342, 199

Corbelli, E., \& Schneider, S. E. 1997, ApJ, 479, 244

Cornwell, T. J. 2008, IEEE J. Select. Topics Signal Process., 2, 793

Deul, E. R., \& van der Hulst, J. M. 1987, A\&AS, 67, 509

Dickman, R. L., Snell, R. L., \& Schloerb, F. P. 1986, ApJ, 309, 326

Digel, S. W., Lyder, D. A., Philbrick, A. J., Puche, D., \& Thaddeus, P. 1996, ApJ, 458,561

Engargiola, G., Plambeck, R. L., Rosolowsky, E., \& Blitz, L. 2003, ApJS, 149, 343

Fukui, Y., Kawamura, A., Minamidani, T., et al. 2008, ApJS, 178, 56

Galleti, S., Bellazzini, M., \& Ferraro, F. R. 2004, A\&A, 423, 925

Gardan, E., Braine, J., Schuster, K. F., Brouillet, N., \& Sievers, A. 2007, A\&A, 473,91

Gratier, P., Braine, J., Rodriguez-Fernandez, N. J., et al. 2010, A\&A, 512, A68 Greenawalt, B. E. 1998, Ph.D. thesis, New Mexico State University Heyer, M. H., Corbelli, E., Schneider, S. E., \& Young, J. S. 2004, ApJ, 602, 723 Hinz, J. L., Rieke, G. H., Gordon, K. D., et al. 2004, ApJS, 154, 259 Hippelein, H., Haas, M., Tuffs, R. J., et al. 2003, A\&A, 407, 137 
Hoopes, C. G., Walterbos, R. A. M., \& Bothun, G. D. 2001, ApJ, 559, 878 Israel, F. 2000, in Molecular Hydrogen in Space, ed. F. Combes, \& G. Pineau Des Forets, 293

Israel, F. P., Baas, F., Rudy, R. J., Skillman, E. D., \& Woodward, C. E. 2003, A\&A, 397, 87

Jorsater, S., \& van Moorsel, G. A. 1995, AJ, 110, 2037

Kennicutt, Jr., R. C. 1998, ApJ, 498, 541

Kramer, C., Boquien, M. X. M., Buchbender, C., et al. 2010, A\&A, 518, L67

Krumholz, M. R., McKee, C. F., \& Tumlinson, J. 2009, ApJ, 699, 850

Leroy, A., Bolatto, A., Walter, F., \& Blitz, L. 2006, ApJ, 643, 825

Leroy, A., Cannon, J., Walter, F., Bolatto, A., \& Weiss, A. 2007, ApJ, 663, 990

Leroy, A. K., Walter, F., Brinks, E., et al. 2008, AJ, 136, 2782

Loinard, L., Dame, T. M., Heyer, M. H., Lequeux, J., \& Thaddeus, P. 1999, A\&A, 351, 1087

Madau, P., Ferguson, H. C., Dickinson, M. E., et al. 1996, MNRAS, 283, 1388

Magrini, L., Stanghellini, L., \& Villaver, E. 2009, ApJ, 696, 729

Murgia, M., Crapsi, A., Moscadelli, L., \& Gregorini, L. 2002, A\&A, 385, 412

Nieten, C., Neininger, N., Guélin, M., et al. 2006, A\&A, 453, 459
Paturel, G., Petit, C., Prugniel, P., et al. 2003, VizieR Online Data Catalog, 7237 Putman, M. E., Peek, J. E. G., Muratov, A., et al. 2009, ApJ, 703, 1486 Rich, J. W., de Blok, W. J. G., Cornwell, T. J., et al. 2008, AJ, 136, 2897 Rohlfs, K., \& Wilson, T. L. 1996, Tools of Radio Astronomy (Springer) Rosolowsky, E., \& Simon, J. D. 2008, ApJ, 675, 1213

Rosolowsky, E., Engargiola, G., Plambeck, R., \& Blitz, L. 2003, ApJ, 599, 258

Rosolowsky, E., Keto, E., Matsushita, S., \& Willner, S. P. 2007, ApJ, 661, 830

Sawada, T., Hasegawa, T., Handa, T., et al. 2001, ApJS, 136, 189

Schuster, K.-F., Boucher, C., Brunswig, W., et al. 2004, A\&A, 423, 1171

Tabatabaei, F. S., Beck, R., Krügel, E., et al. 2007a, A\&A, 475, 133

Tabatabaei, F. S., Krause, M., \& Beck, R. 2007b, A\&A, 472, 785

Thilker, D. A., Hoopes, C. G., Bianchi, L., et al. 2005, ApJ, 619, L67

Verley, S., Hunt, L. K., Corbelli, E., \& Giovanardi, C. 2007, A\&A, 476, 1161

Verley, S., Corbelli, E., Giovanardi, C., \& Hunt, L. K. 2009, A\&A, 493, 453

Wilkins, S. M., Trentham, N., \& Hopkins, A. M. 2008, MNRAS, 385, 687

Wilson, C. D. 1995, ApJ, 448, L97

Wilson, C. D., \& Scoville, N. 1990, ApJ, 363, 435

Wilson, C. D., Walker, C. E., \& Thornley, M. D. 1997, ApJ, 483, 210

Pages 15 to 29 are available in the electronic edition of the journal at http://www . aanda.org 
P. Gratier et al.: Molecular and atomic gas in the Local Group galaxy M 33

Appendix A: Online figures

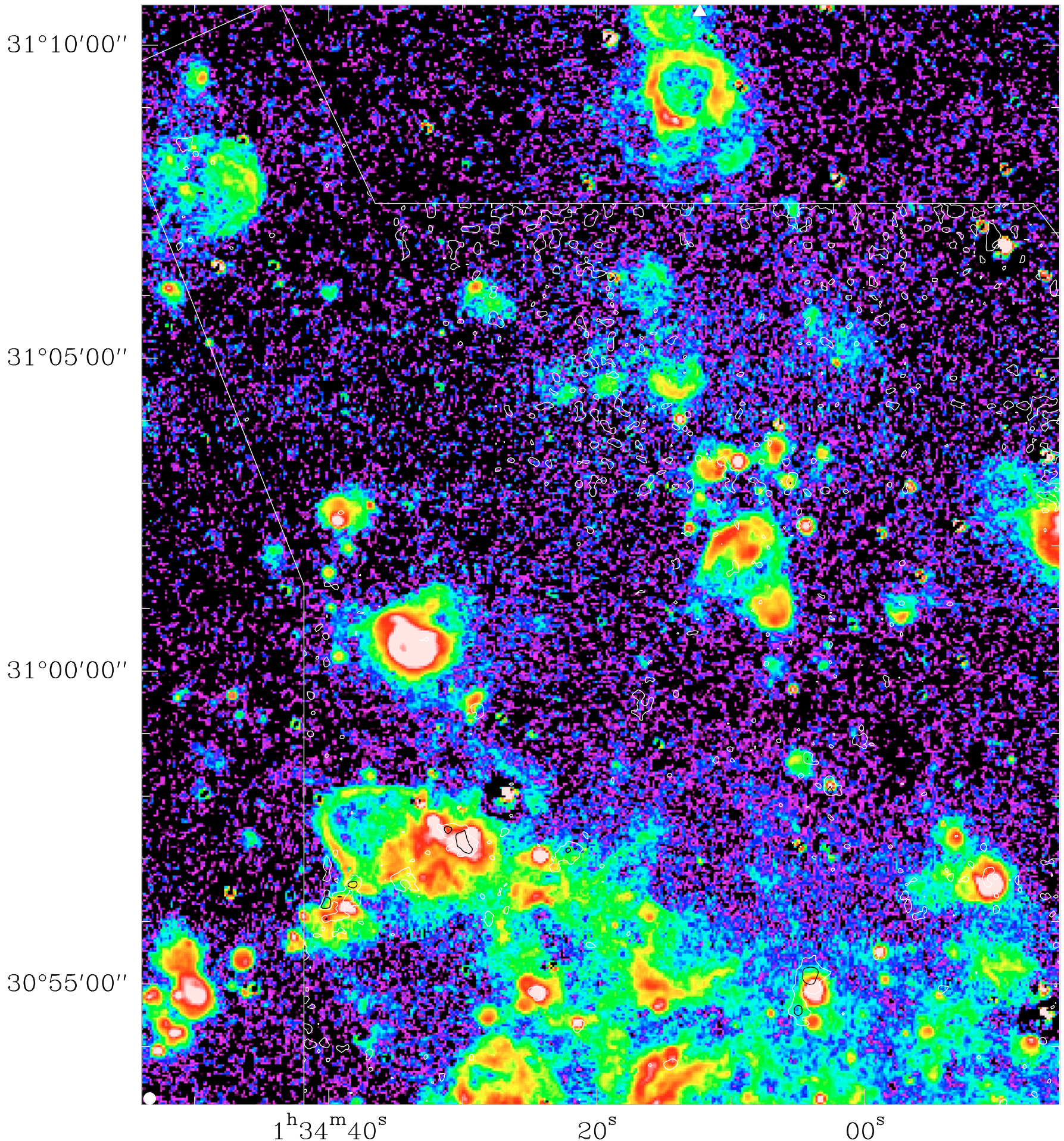

Fig. A.1. Northern part of KPNO $\mathrm{H} \alpha$ image with $I_{\mathrm{CO}}$ main beam contours of 1 (white), 2, 4 (black), 8 (blue) $\mathrm{K} \mathrm{km} \mathrm{s}^{-1}$. The beam size is shown as a white dot in the lower left corner. 


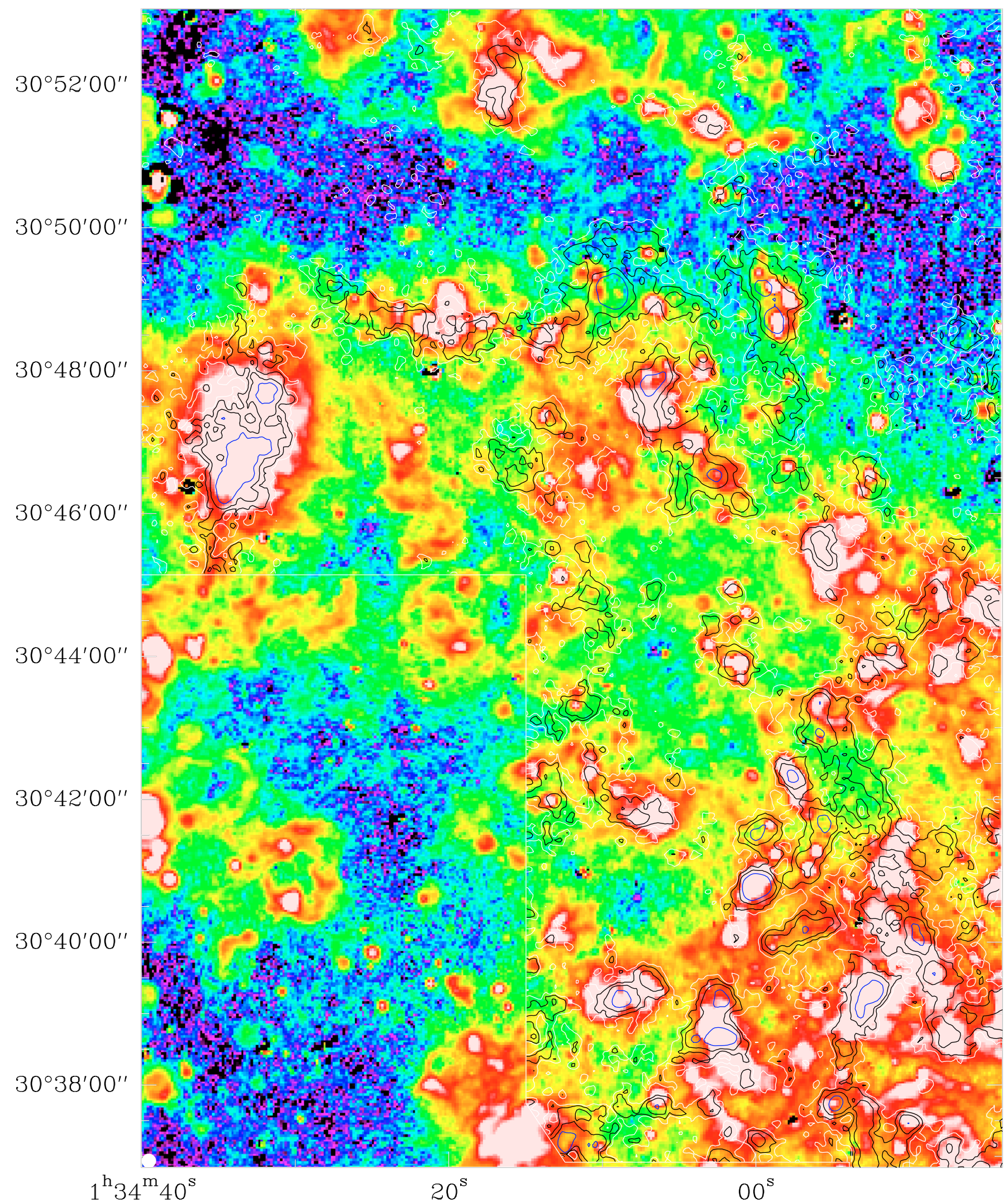

Fig. A.2. Center part of KPNO $\mathrm{H} \alpha$ image with $I_{\mathrm{CO}}$ main beam contours of 1 (white), 2, 4 (black), 8 (blue) $\mathrm{K} \mathrm{km} \mathrm{s}^{-1}$. The beam size is shown as a white dot in the lower left corner. 
P. Gratier et al.: Molecular and atomic gas in the Local Group galaxy M 33

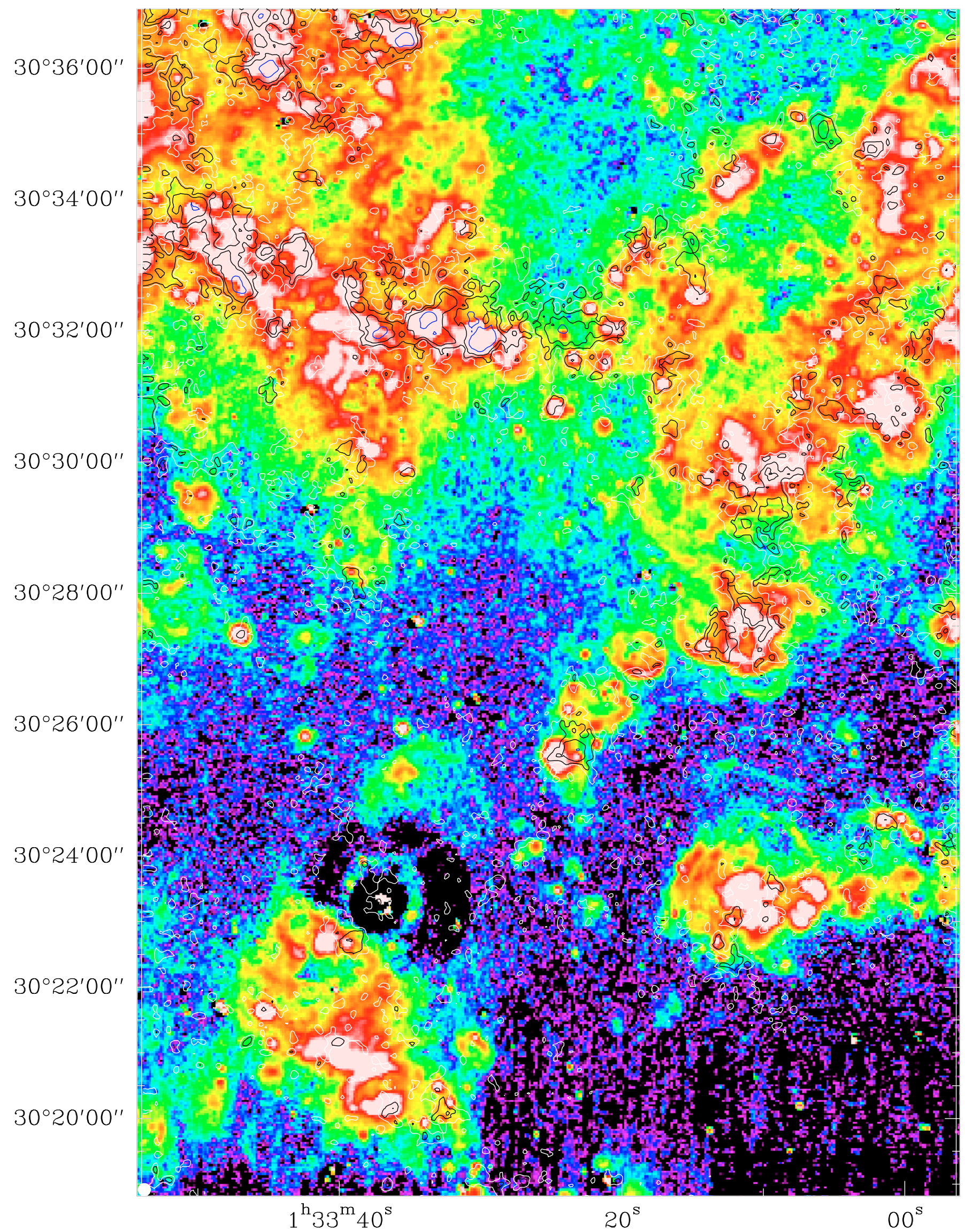

Fig. A.3. Southern part of KPNO H $\alpha$ image with $I_{\mathrm{CO}}$ main beam contours of 1 (white), 2, 4 (black), 8 (blue) $\mathrm{K} \mathrm{km} \mathrm{s}{ }^{-1}$. The beam size is shown as a white dot in the lower left corner. 
A\&A 522, A3 (2010)

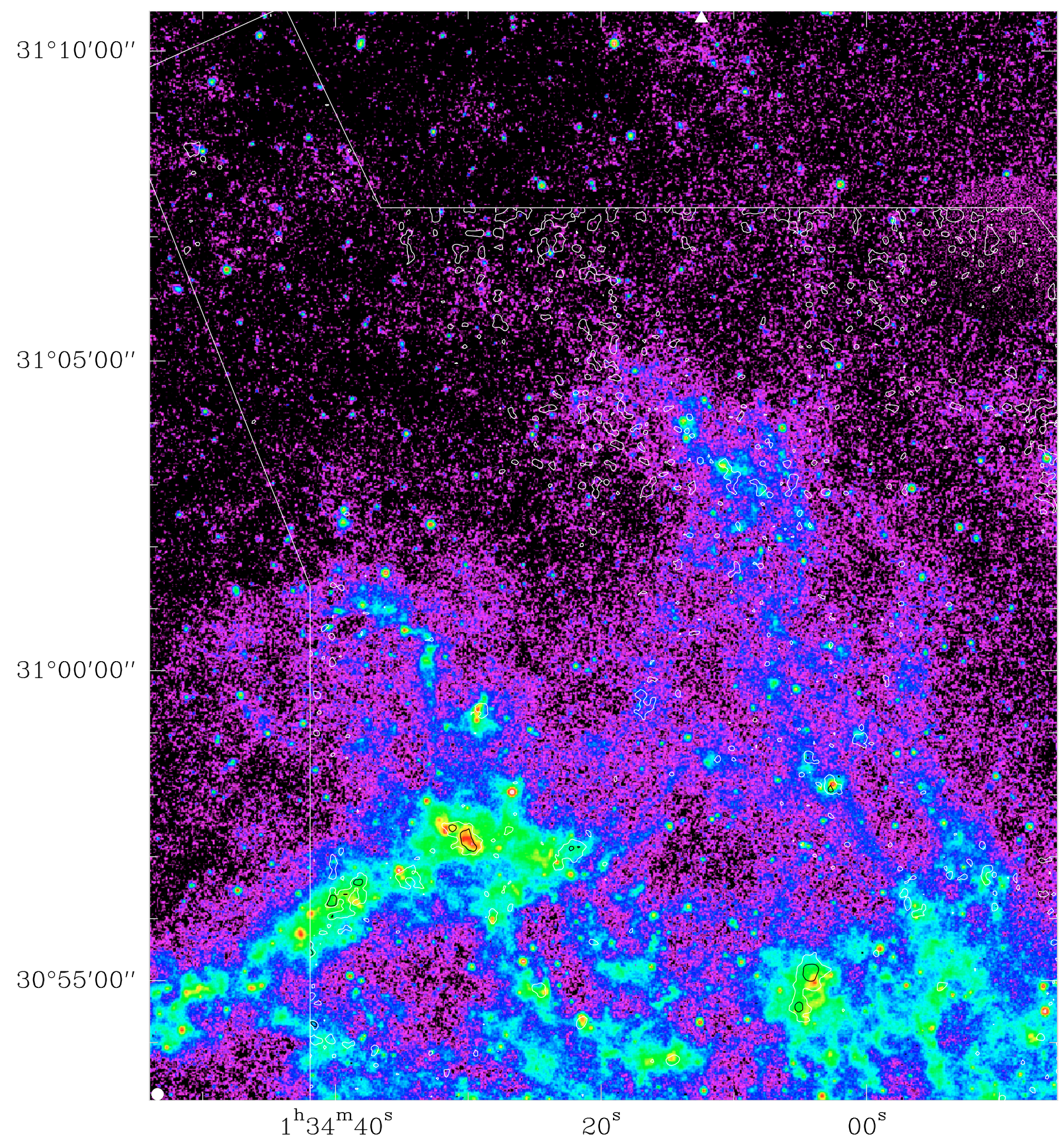

Fig. A.4. Northern part of Spitzer $8 \mu \mathrm{m}$ image with $I_{\mathrm{CO}}$ main beam contours of 1 (white), 2, 4 (black), 8 (blue) $\mathrm{K} \mathrm{km} \mathrm{s}^{-1}$. The beam size is shown as a white dot in the lower left corner. 
P. Gratier et al.: Molecular and atomic gas in the Local Group galaxy M 33

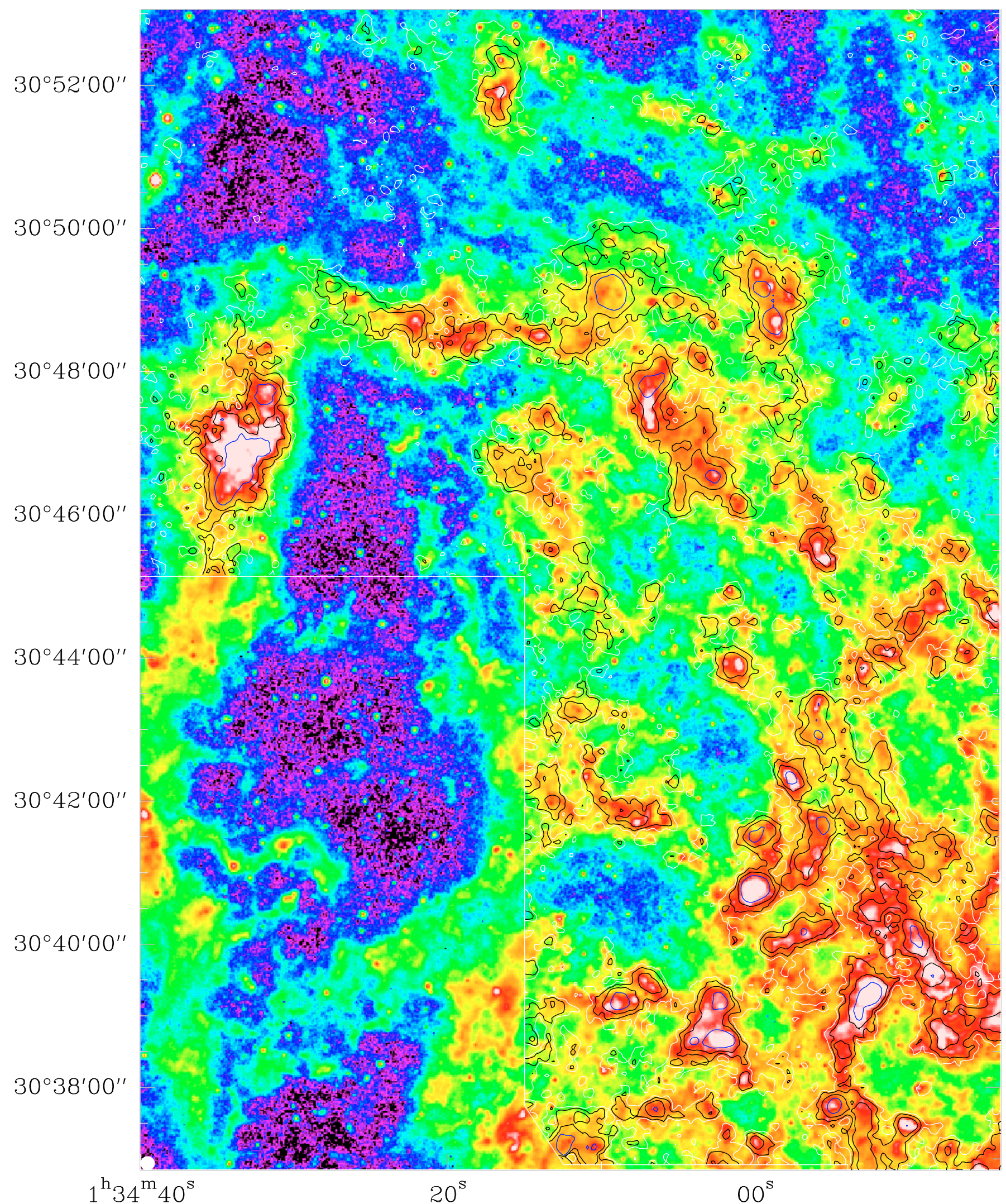

Fig. A.5. Center part of Spitzer $8 \mu \mathrm{m}$ image with $I_{\mathrm{CO}}$ main beam contours of 1 (white), 2, 4 (black), 8 (blue) $\mathrm{K} \mathrm{km} \mathrm{s}^{-1}$. The beam size is shown as a white dot in the lower left corner. 


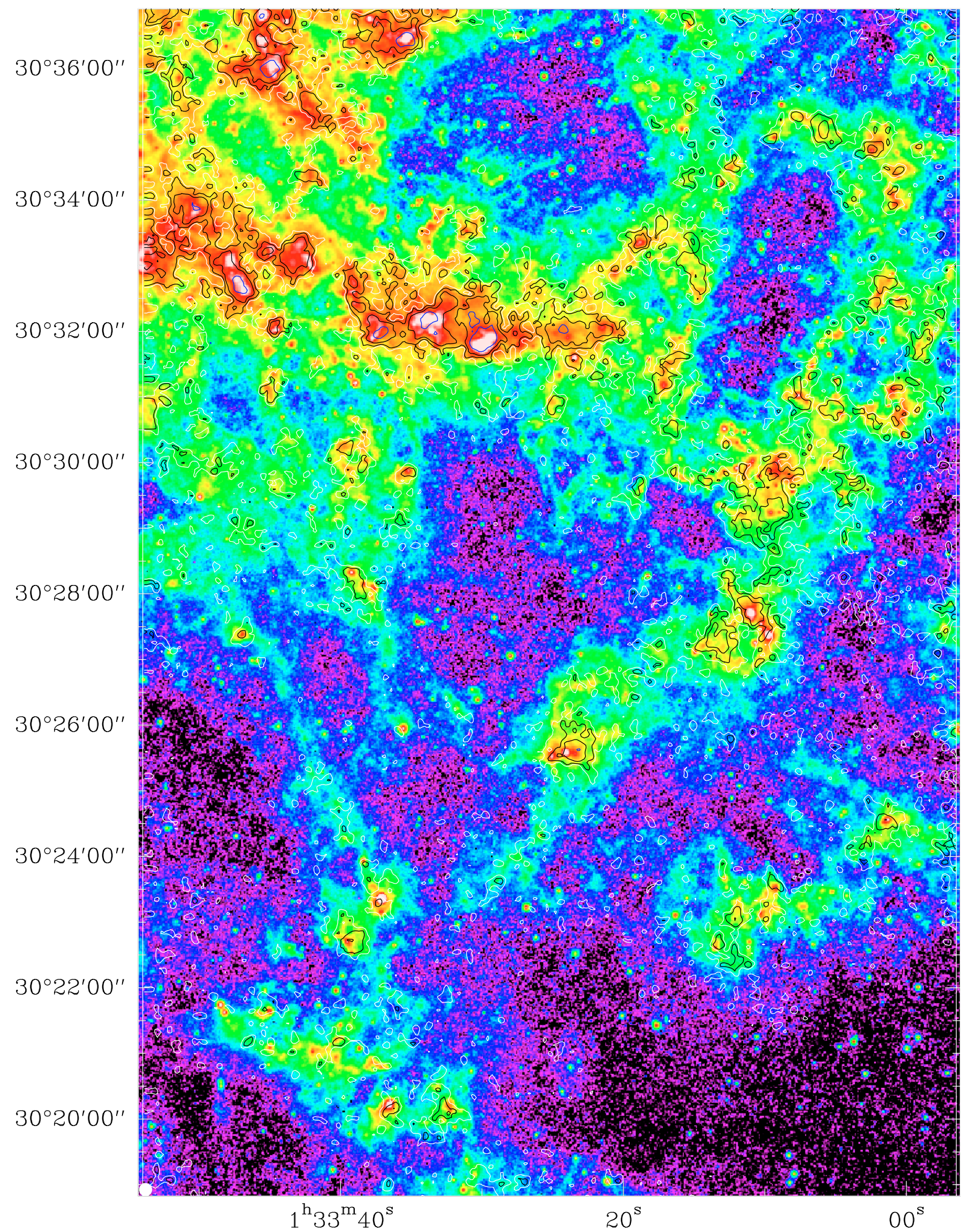

Fig. A.6. Southern part of Spitzer $8 \mu \mathrm{m}$ image with $I_{\mathrm{CO}}$ main beam contours of 1 (white), 2, 4 (black), 8 (blue) $\mathrm{K} \mathrm{km} \mathrm{s}^{-1}$. The beam size is shown as a white dot in the lower left corner. 


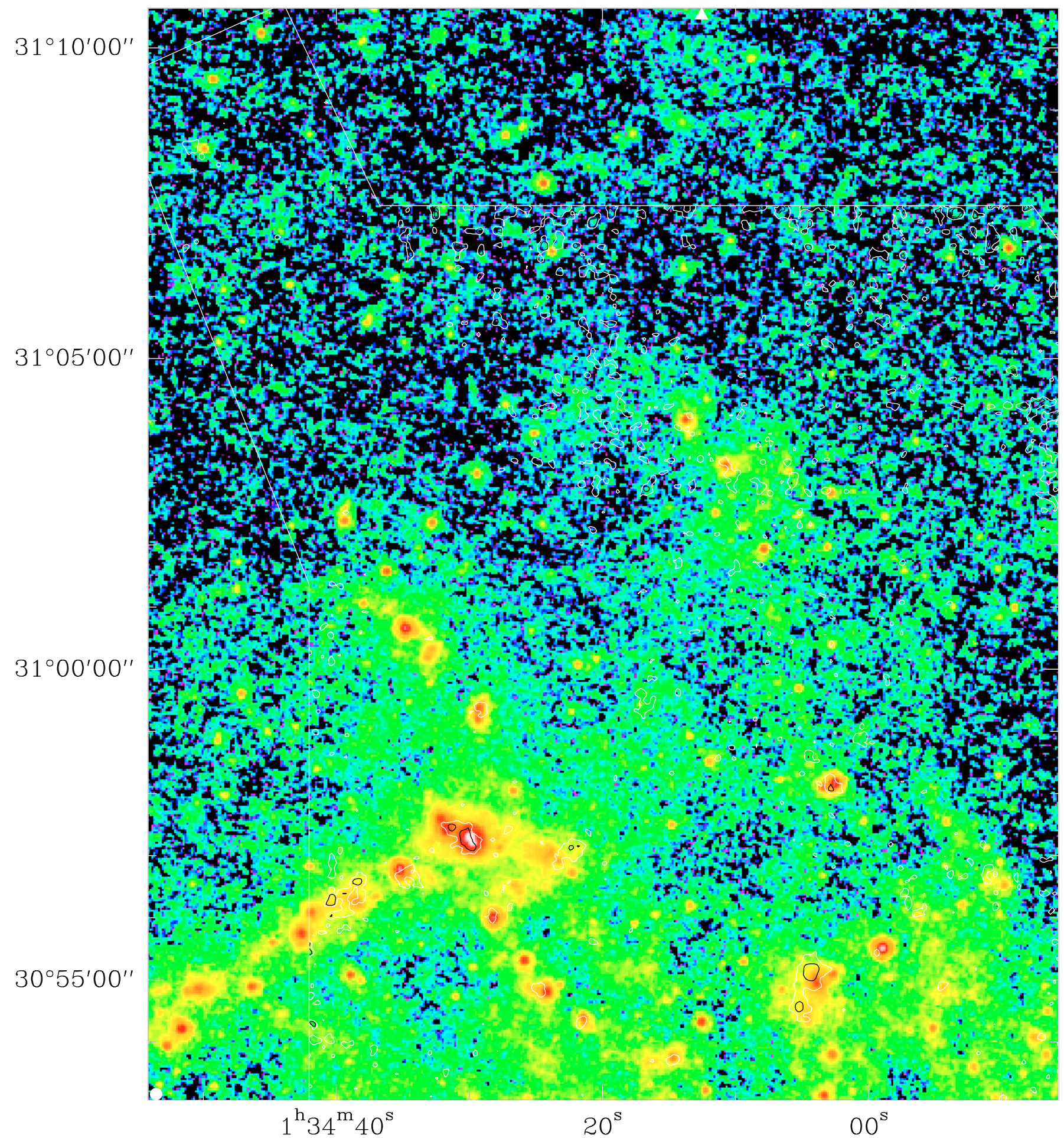

Fig. A.7. Northern part of Spitzer $24 \mu \mathrm{m}$ image with $I_{\mathrm{CO}}$ main beam contours of 1 (white), 2, 4 (black), 8 (blue) $\mathrm{K} \mathrm{km} \mathrm{s}^{-1}$. The beam size is shown as a white dot in the lower left corner. 


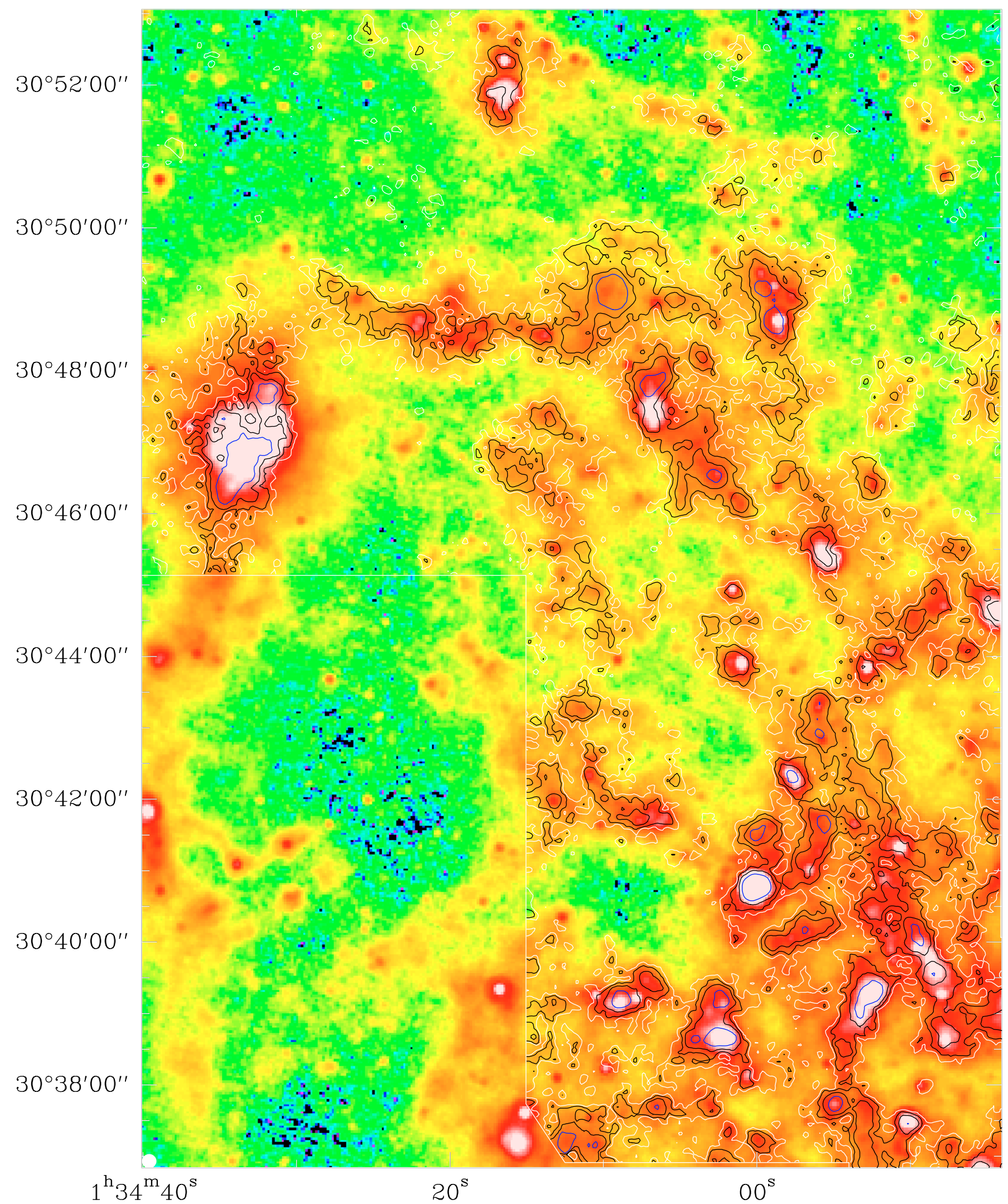

Fig. A.8. Center part of Spitzer $24 \mu \mathrm{m}$ image with $I_{\mathrm{CO}}$ main beam contours of 1 (white), 2, 4 (black), 8 (blue) $\mathrm{K} \mathrm{km} \mathrm{s}^{-1}$. The beam size is shown as a white dot in the lower left corner. 
P. Gratier et al.: Molecular and atomic gas in the Local Group galaxy M 33

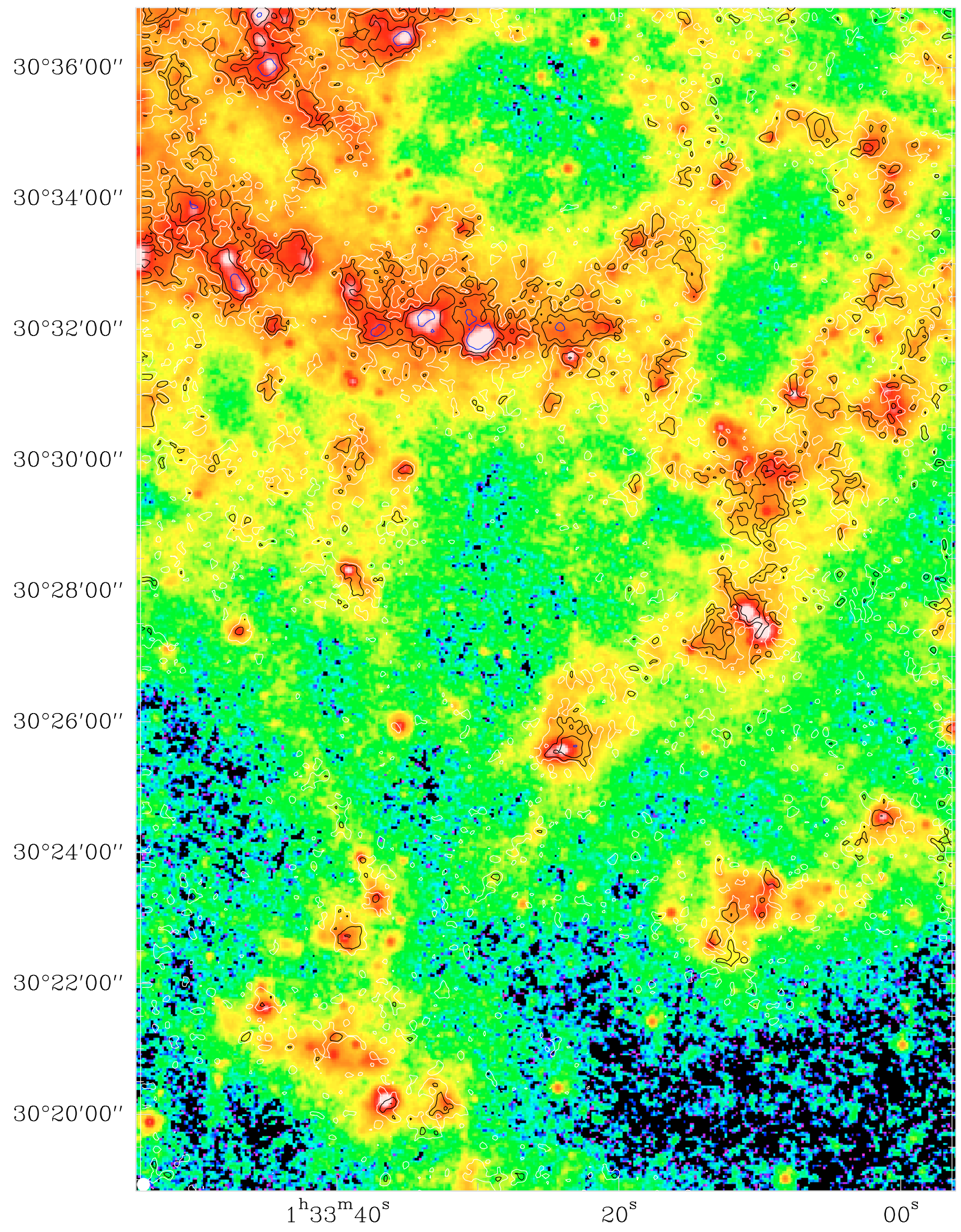

Fig. A.9. Southern part of Spitzer $24 \mu \mathrm{m}$ image with $I_{\mathrm{CO}}$ main beam contours of 1 (white), 2, 4 (black), 8 (blue) $\mathrm{K} \mathrm{km} \mathrm{s}^{-1}$. The beam size is shown as a white dot in the lower left corner. 


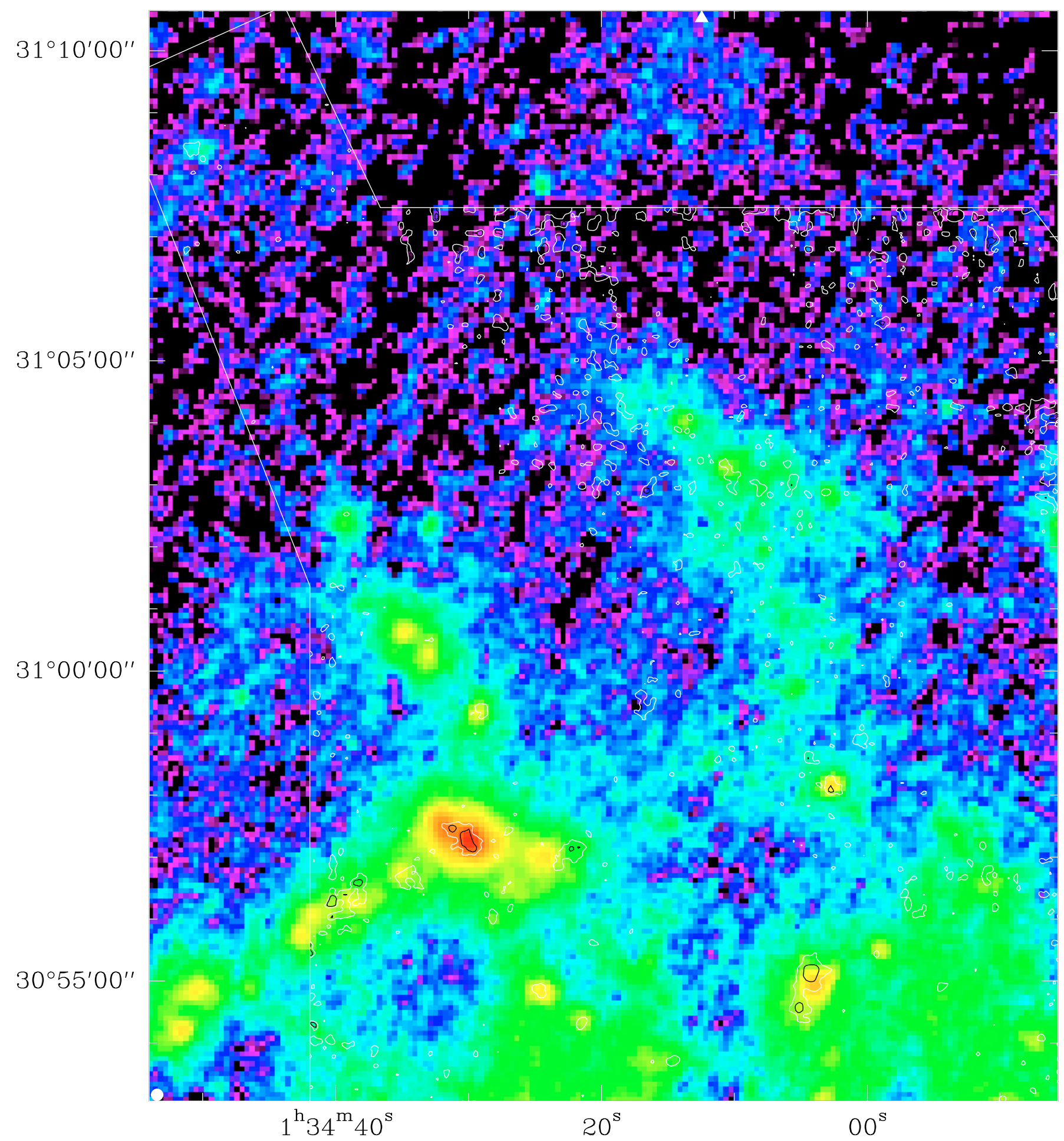

Fig. A.10. Northern part of Spitzer $70 \mu \mathrm{m}$ image with $I_{\mathrm{CO}}$ main beam contours of 1 (white), 2, 4 (black), 8 (blue) $\mathrm{K} \mathrm{km} \mathrm{s}^{-1}$. The beam size is shown as a white dot in the lower left corner. 
P. Gratier et al.: Molecular and atomic gas in the Local Group galaxy M 33

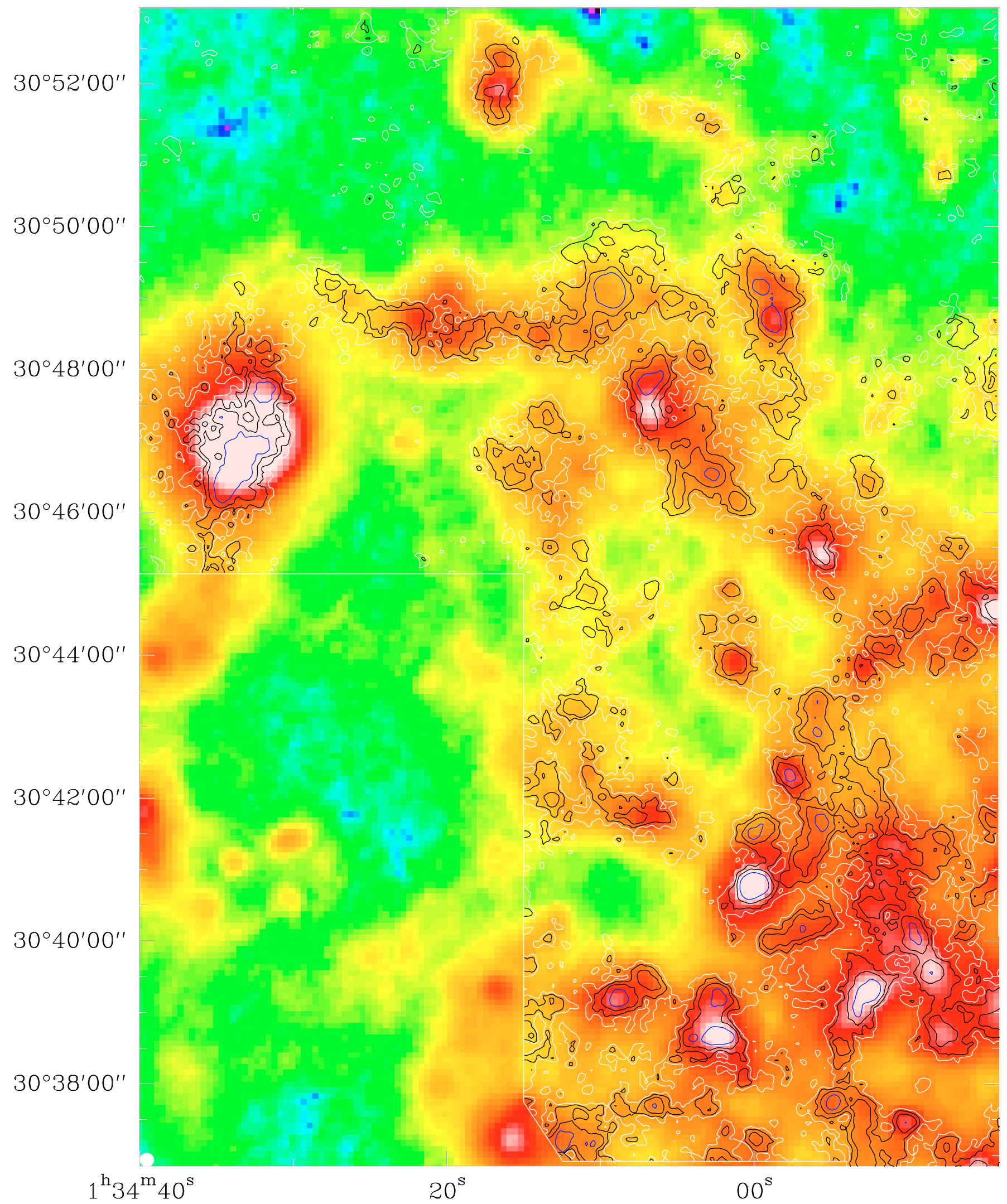

Fig. A.11. Center part of Spitzer $70 \mu \mathrm{m}$ image with $I_{\mathrm{CO}}$ main beam contours of 1 (white), 2, 4 (black), 8 (blue) $\mathrm{K} \mathrm{km} \mathrm{s}^{-1}$. The beam size is shown as a white dot in the lower left corner. 


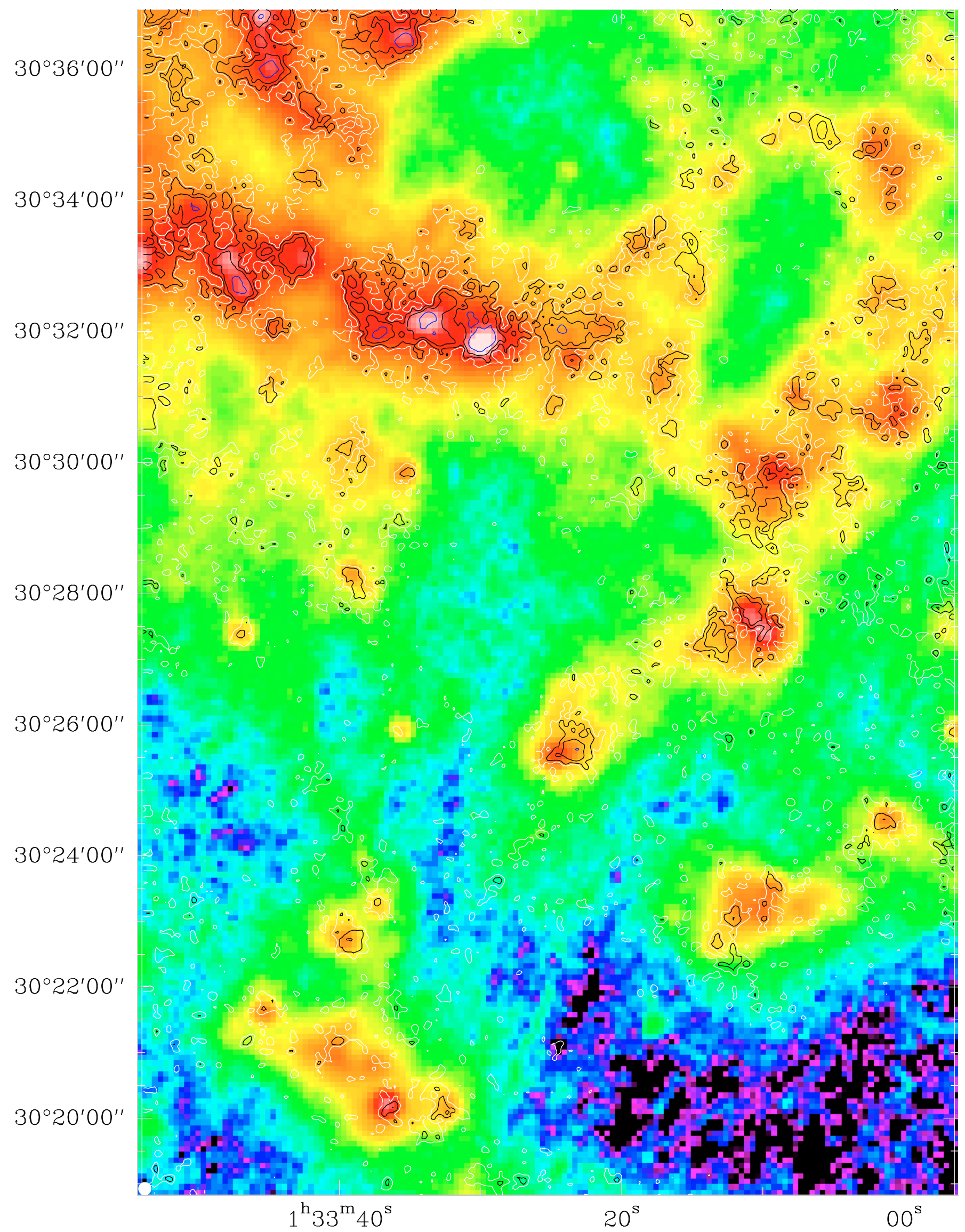

Fig. A.12. Southern part of Spitzer $70 \mu \mathrm{m}$ image with $I_{\mathrm{CO}}$ main beam contours of 1 (white), 2, 4 (black), 8 (blue) $\mathrm{K} \mathrm{km} \mathrm{s}^{-1}$. The beam size is shown as a white dot in the lower left corner. 
P. Gratier et al.: Molecular and atomic gas in the Local Group galaxy M 33

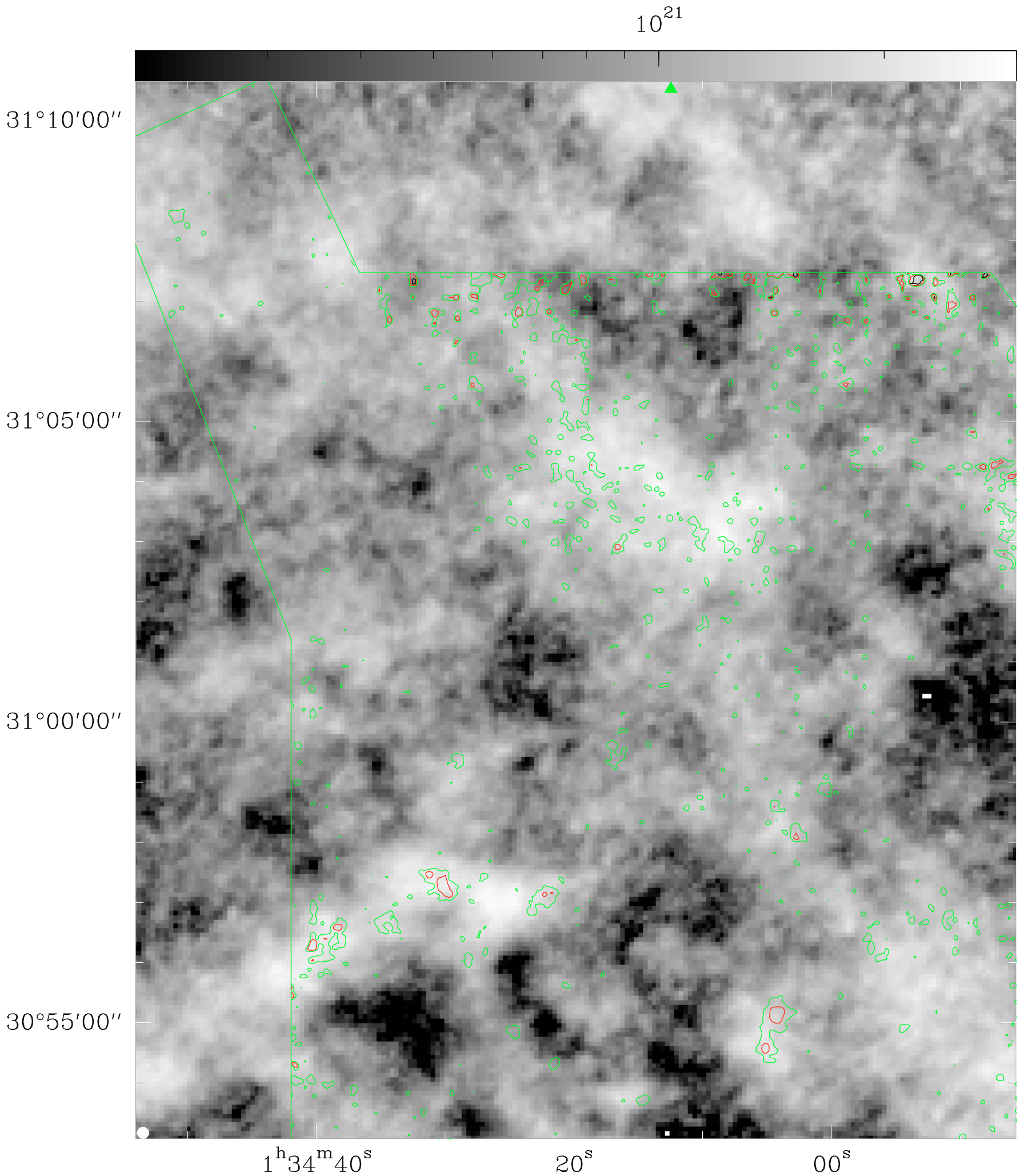

Fig. A.13. Northern part of VLA H I $21 \mathrm{~cm}$ integrated intensity image with $I_{\mathrm{CO}}$ main beam contours of 1 (green), 2 (red), 4(black), 8 (blue) $\mathrm{K} \mathrm{km} \mathrm{s}^{-1}$. The beam size is shown as a white dot in the lower left corner. 


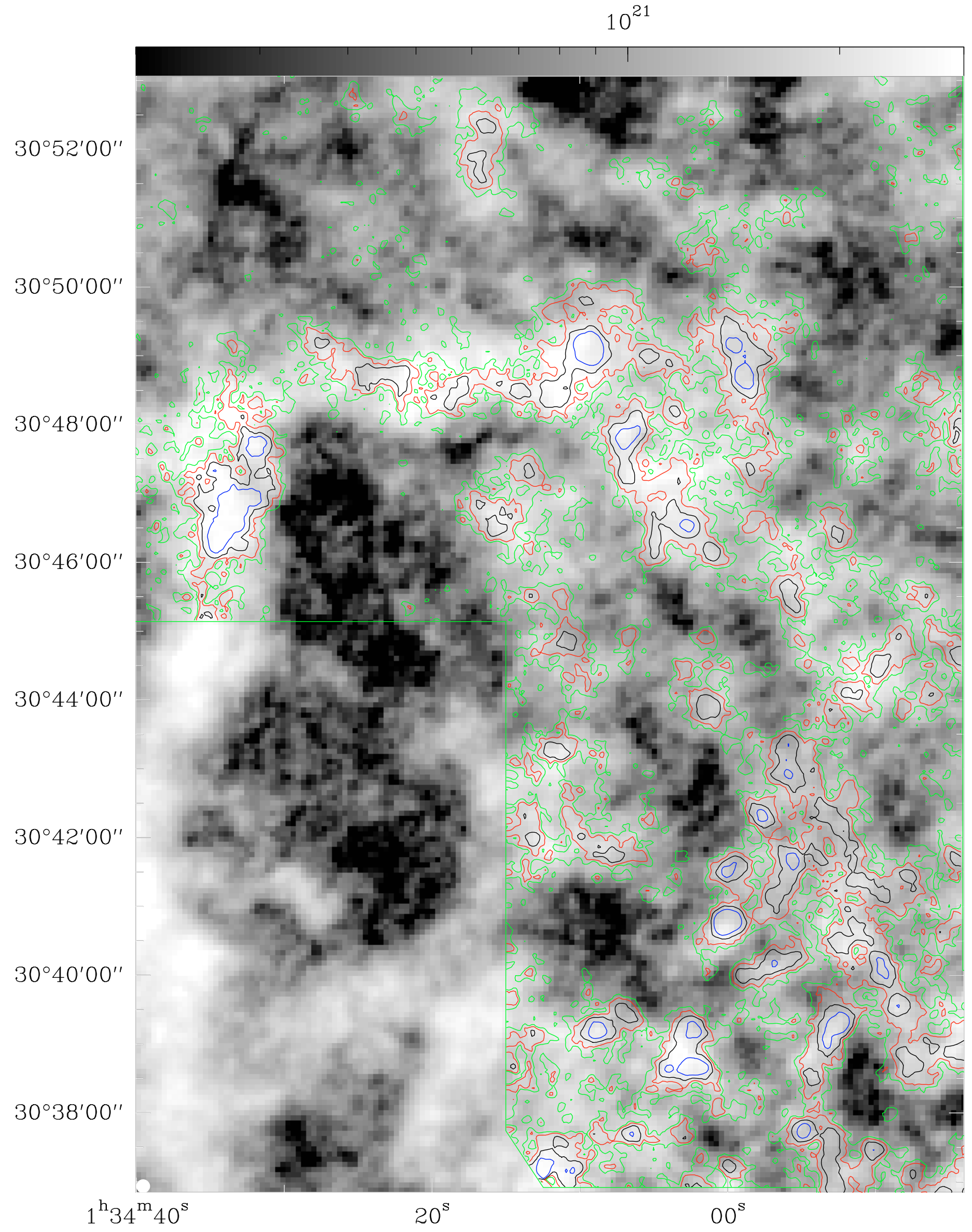

Fig. A.14. Center part of VLA H I $21 \mathrm{~cm}$ integrated intensity image with $I_{\mathrm{CO}}$ main beam contours of 1 (green), 2 (red), 4(black), 8 (blue) K km s ${ }^{-1}$. The beam size is shown as a white dot in the lower left corner. 
P. Gratier et al.: Molecular and atomic gas in the Local Group galaxy M 33

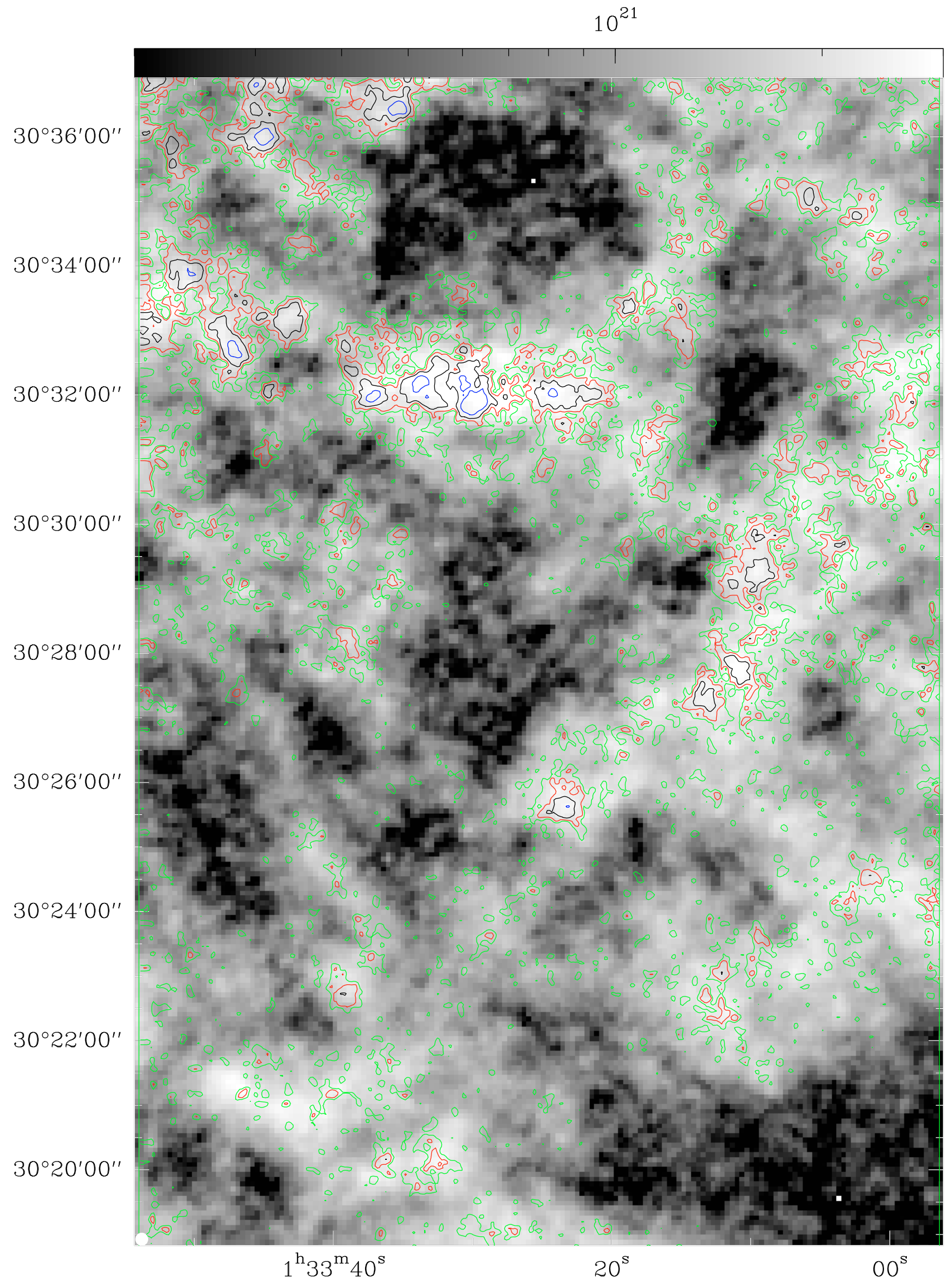

Fig. A.15. Southern part of VLA H I $21 \mathrm{~cm}$ integrated intensity image with $I_{\mathrm{CO}}$ main beam contours of 1 (green), 2 (red), 4(black), 8 (blue) $\mathrm{K} \mathrm{km} \mathrm{s}^{-1}$. The beam size is shown as a white dot in the lower left corner. 